\title{
Initial Calibration of the Atmospheric Imaging Assembly (AIA) on the Solar Dynamics Observatory (SDO)
}

\author{
Paul Boerner • Christopher Edwards • James Lemen • Adam Rausch • \\ Carolus Schrijver • Richard Shine • Lawrence Shing • Robert Stern • \\ Theodore Tarbell • Alan Title • C. Jacob Wolfson • Regina Soufli • Eberhard Spiller • \\ Eric Gullikson • David McKenzie • David Windt • Leon Golub • William Podgorski • \\ Paola Testa $\cdot$ Mark Weber
}

Received: 8 September 2010 / Accepted: 24 May 2011 / Published online: 22 July 2011

(C) The Author(s) 2011. This article is published with open access at Springerlink.com

\begin{abstract}
The Atmospheric Imaging Assembly (AIA) instrument onboard the Solar Dynamics Observatory (SDO) is an array of four normal-incidence reflecting telescopes that image the Sun in ten EUV and UV wavelength channels. We present the initial photometric calibration of AIA, based on preflight measurements of the response of the telescope components. The estimated accuracy is of order $25 \%$, which is consistent with the results of comparisons with full-disk irradiance measurements and spectral models. We also describe the characterization of the instrument performance, including image resolution, alignment, camera-system gain, flat-fielding, and data compression.
\end{abstract}

Keywords Instrumentation $\cdot$ EUV $\cdot$ Soft X-ray $\cdot$ Chromosphere $\cdot$ Corona $\cdot$ Transition region

The Solar Dynamics Observatory

Guest Editors: W. Dean Pesnell, Phillip C. Chamberlin, and Barbara J. Thompson

P. Boerner $(\varangle) \cdot$ C. Edwards · J. Lemen · A. Rausch · C. Schrijver · R. Shine · L. Shing · R. Stern ·

T. Tarbell $\cdot$ A. Title $\cdot$ C.J. Wolfson

Solar and Astrophysics Laboratory, Dept ADBS Bldg 252, Lockheed Martin Advanced Technology

Center, 3251 Hanover St., Palo Alto, CA 94304, USA

e-mail: boerner@Imsal.com

R. Soufli · E. Spiller

Lawrence Livermore National Laboratory, Livermore, CA, USA

E. Gullikson

Lawrence Berkeley National Laboratory, Berkeley, CA, USA

D. McKenzie

Montana State University, Bozeman, MT, USA

D. Windt

Reflective X-ray Optics LLC, New York, NY, USA

L. Golub · W. Podgorski · P. Testa · M. Weber

Smithsonian Astrophysical Observatory, Cambridge, MA, USA 


\section{Introduction}

The Atmospheric Imaging Assembly (AIA) instrument onboard the Solar Dynamics Observatory (SDO) explores the structure and dynamics of the solar atmosphere with unprecedented cadence and coverage, both spatial and spectral. It has operated essentially continuously since 28 April 2010. AIA is described in detail in Lemen et al. (2011); the data processing approach is described by Hurlburt et al. (2011). It consists of an array of four dualchannel normal-incidence telescopes, which observe an 41-arcmin field of view in seven EUV and three UV-visible-light channels with 0.6-arcsec pixels on $4096 \times 4096$ CCDs. In the normal observing mode, each camera records an image every 5-6 seconds, so that each of the EUV channels is imaged every 10-12 seconds (the UV/visible channels are imaged at a lower cadence). In this article, we describe the initial calibration of AIA, including the photometric calibration that is required to perform quantitative analysis of the AIA data, and characterization of the instrument parameters that affect the data products.

\section{Photometric Calibration}

The basic AIA data product consists of an image of pixel values $p_{i}(\mathbf{x})$, where the index $i$ refers to one of the ten wavelength channels and $\mathbf{x}$ refers to a particular position in the image plane. These pixel values are measurements of the solar spectral radiance integrated over the solid angle subtended by the pixel and the wavelength passband of the telescope channel:

$$
p_{i}(\mathbf{x})=\int_{0}^{\infty} \eta_{i}(\lambda) \mathrm{d} \lambda \int_{\text {pixel } \mathbf{x}} I(\lambda, \boldsymbol{\theta}) \mathrm{d} \boldsymbol{\theta} .
$$

Here $\eta_{i}$ is the efficiency function of the $i$ th channel of the telescope - its passband, in units of digital number [DN] per unit flux at the aperture. It can be calculated as follows:

$$
\eta(\lambda, t, \mathbf{x})=A_{\mathrm{eff}}(\lambda, t) G(\lambda) F(\mathbf{x}),
$$

where $G$ is the gain of the CCD-camera system in DN per photon - it combines the standard conversion of photons to detected electrons with the camera gain $[g]$, in DN per electron:

$$
G(\lambda)=(12398 / \lambda / 3.65) g
$$

The field-angle (position in the image) dependent factors, including vignetting, filter grid shadowing, and CCD sensitivity variations, are included in the flat-field function $[F]$.

The effective area $\left[A_{\text {eff }}\right]$ contains information about the efficiency of the telescope optics, as follows:

$$
A_{\text {eff }}(\lambda, t)=A_{\text {geo }} R_{\mathrm{P}}(\lambda) R_{\mathrm{S}}(\lambda) T_{\mathrm{E}}(\lambda) T_{\mathrm{F}}(\lambda) D(\lambda, t) Q(\lambda) .
$$

The geometrical collecting area $\left[A_{\text {geo }}\right]$ is multiplied by the reflectance $[R]$ of the primary and secondary mirrors, the transmission efficiency $[T]$ of the entrance and focal-plane filters, the quantum efficiency $[Q]$ of the $\mathrm{CCD}$, and an additional correction $[D]$ to account for the time-varying effect of degradation due to contamination or deterioration of the components.

In practice, calibration of the AIA data proceeds in two directions. Where possible, the data are corrected for instrumental effects, so the vignetting and flat-field variations are divided out of the image data for Level 1 and higher (Hurlburt et al., 2011). However, because the AIA CCDs provide no spectroscopic information, there is no straightforward way to 


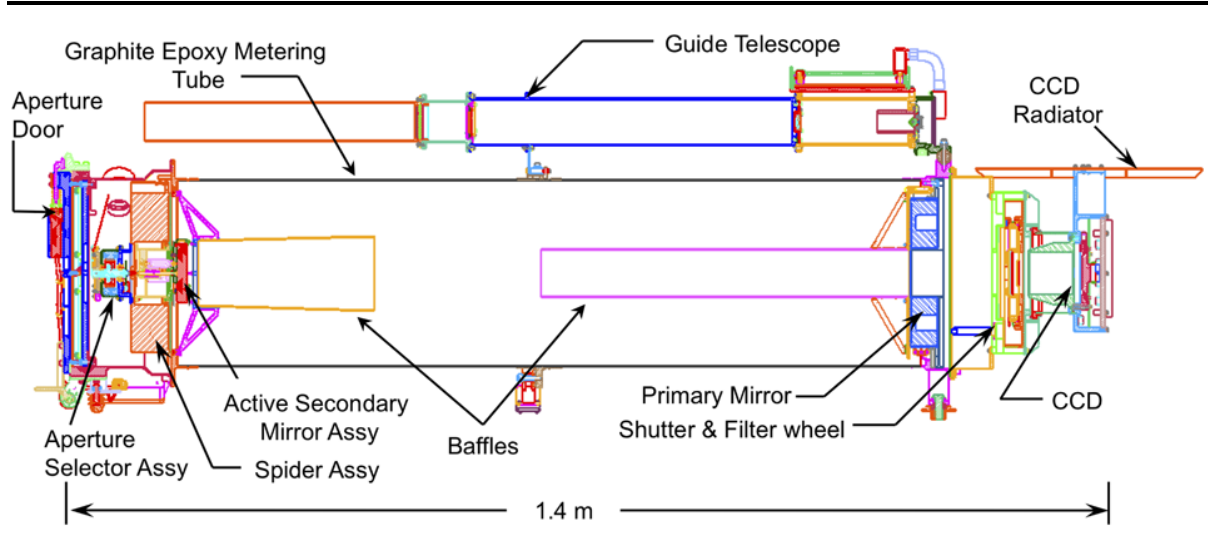

Figure 1 Schematic layout of an AIA telescope assembly.

apply the wavelength-dependent calibration to the data directly. Therefore the instrumentresponse function $\left[R(\lambda, t)=A_{\text {eff }}(\lambda, t) G(\lambda)\right]$ is provided to the data user and can be incorporated into an analysis of the images.

\subsection{Measuring the Response Function}

Due to the difficulty in obtaining a well-calibrated, narrowband, collimated EUV source, an end-to-end EUV calibration measurement of the AIA response function was not feasible. Instead, the photometric calibration was obtained by making component-level measurements of all the optical elements in the AIA telescopes (mirrors, filters, and CCD), and combining those measurements analytically to produce a model of the system performance. This approach was used in the calibration of TRACE (Handy et al., 1999) and EIT (Dere et al., 2000) as well. The optical elements are shown schematically in Figure 1.

\subsubsection{Geometric Area}

The AIA telescope assemblies (ATAs) are 20-cm aperture Ritchey-Chrétien optical systems whose apertures have been divided in half by applying different coatings to the top and bottom sectors of the mirrors. For each exposure, one half of the aperture is selected either by blocking the other half with a motorized shutter at the aperture, or by selecting a focal-plane filter that rejects photons at the other channel's wavelength. The coated areas of the mirrors are masked in order to prevent reflections from one half of the primary mirror reaching the opposite half of the secondary. A semi-annular mask on the primary mirror defines the pupil of the system. The geometric collecting area is further limited by obstruction due to the secondary mirror and the structural elements of the telescope spider and entrance-filter supports. The geometric area of each EUV channel is $83.0 \mathrm{~cm}^{2}$.

The UV channel does not make use of the full semi-annulus of open area in the primarymirror mask; its aperture is stopped down by a roughly circular mask in front of the UV window. The geometric area of the UV channel is $30.8 \mathrm{~cm}^{2}$. The filters and mirrors in the UV channel are qualitatively different from the EUV filters and mirrors; therefore, they are discussed in Section 2.1.5 after the discussion of the EUV channel filters and mirrors. 
Table 1 Parameters of the models used to fit the measurements of the thin-film metal filters.

\begin{tabular}{ll}
\hline Filter & Model \\
\hline $\mathrm{Al}$ thin/Al entrance & $1450 \AA \mathrm{Al}+87 \AA \mathrm{Al}_{2} \mathrm{O}_{3}$ \\
$\mathrm{Al}$ thick & $2450 \AA \mathrm{Al}+103 \AA \mathrm{Al}_{2} \mathrm{O}_{3}$ \\
$\mathrm{Zr}$ thin/Zr entrance & $2160 \AA \mathrm{Zr}+199 \AA \mathrm{ZrO}_{2}$ \\
$\mathrm{Zr}$ thick & $3236 \AA \mathrm{Zr}+171 \AA \mathrm{ZrO}_{2}$ \\
$\mathrm{Zr}$ on polyimide & $4000 \AA$ Poly $+2570 \AA \mathrm{Zr}+267 \AA \mathrm{ZrO}_{2}$ \\
\hline
\end{tabular}

\subsubsection{EUV Filters}

AIA's EUV channels use thin-film metal filters mounted on an electroformed nickel mesh to reject visible and IR radiation. The mesh transmission is $82-84 \%$ (this factor is included in the filter transmission and not in the geometric collecting area, although it acts similarly). The filters were manufactured by Luxel Corporation. Most channels use Al filters; the two short-wavelength channels, below the Al L edge (94 $\AA$ and $131 \AA$ ), use $\mathrm{Zr}$ filters instead. All channels have two focal-plane filters available for their use: a thin one (1500 $\AA \mathrm{Al}$ or $2000 \AA \mathrm{Zr}$ ), which is used the majority of the time, and a thicker one (2500 $\mathrm{Al}$ or $3000 \AA$ $\mathrm{Zr}$ ) which is primarily used as a spare. The thick focal-plane filter for the 131- $\AA$ channel has a $\mathrm{Zr}$ film on a polyimide substrate (rather than a nickel mesh); this provides enhanced attenuation of the signal in the $131-\AA$ channel (necessary because of the extreme dynamic range of the 131- $\AA$ images during flares).

The focal-plane filters were measured using the EUV reflectometer at the Lockheed Martin Solar and Astrophysics Laboratory (LMSAL) (Catura et al., 1987). A Henke X-ray tube or hollow-cathode discharge was used to illuminate a 1-m McPherson grazing-incidence grating monochromator, which selected a single wavelength and sent a pencil beam to illuminate the detector (a proportional counter for $\lambda<200 \AA$, and a microchannel plate crosscalibrated with a National Institute of Standards and Technology (NIST) windowless photodiode for $\lambda>200 \AA$ ). The ratio of the detected signal with and without the filter in place was measured. The results of the filter calibration measurements were used to constrain a model of the filter composition, which was calculated using optical constants taken from the LLNL CXRO database (Henke, Gullikson, and Davis, 1993). The details of the filter model are described in Table 1; the measured and modeled filter transmittance are shown in Figure 2.

The flight focal-plane filters themselves were not measured, but the filter calibration program included a series of measurements on filter samples and flight-like filters from different processing runs. The scatter in repeated measurements on a single filter, or between measurements on different flight-like filters and test coupons from the same run, was $1 \%$ in absolute transmittance. This is slightly larger than the shot noise and is probably due to variation in the source or detector. Measurements on different filter lots with the same prescription agreed to within $1.5 \%$. The focal-plane filter models were also used for the entrance filters, which had the same prescription. The entrance filters were measured for visible-light rejection, but they were not calibrated in-band.

There is some additional uncertainty in applying the model to wavelengths where the optical constants are not well known; in particular, the optical constants for Al change rapidly with wavelength (due to the presence of the $\mathrm{Al} \mathrm{L}_{2,3}$ absorption edge) in the vicinity of the Fe IX line targeted by the $171-\AA$ channel, so small variations in the assumed Al optical constants can have substantial effects on the predicted intensity of the images in the 171- $\AA$ channel. The overall uncertainty in applying the filter model to the transmission of the flight filters is therefore of order $5 \%$. 


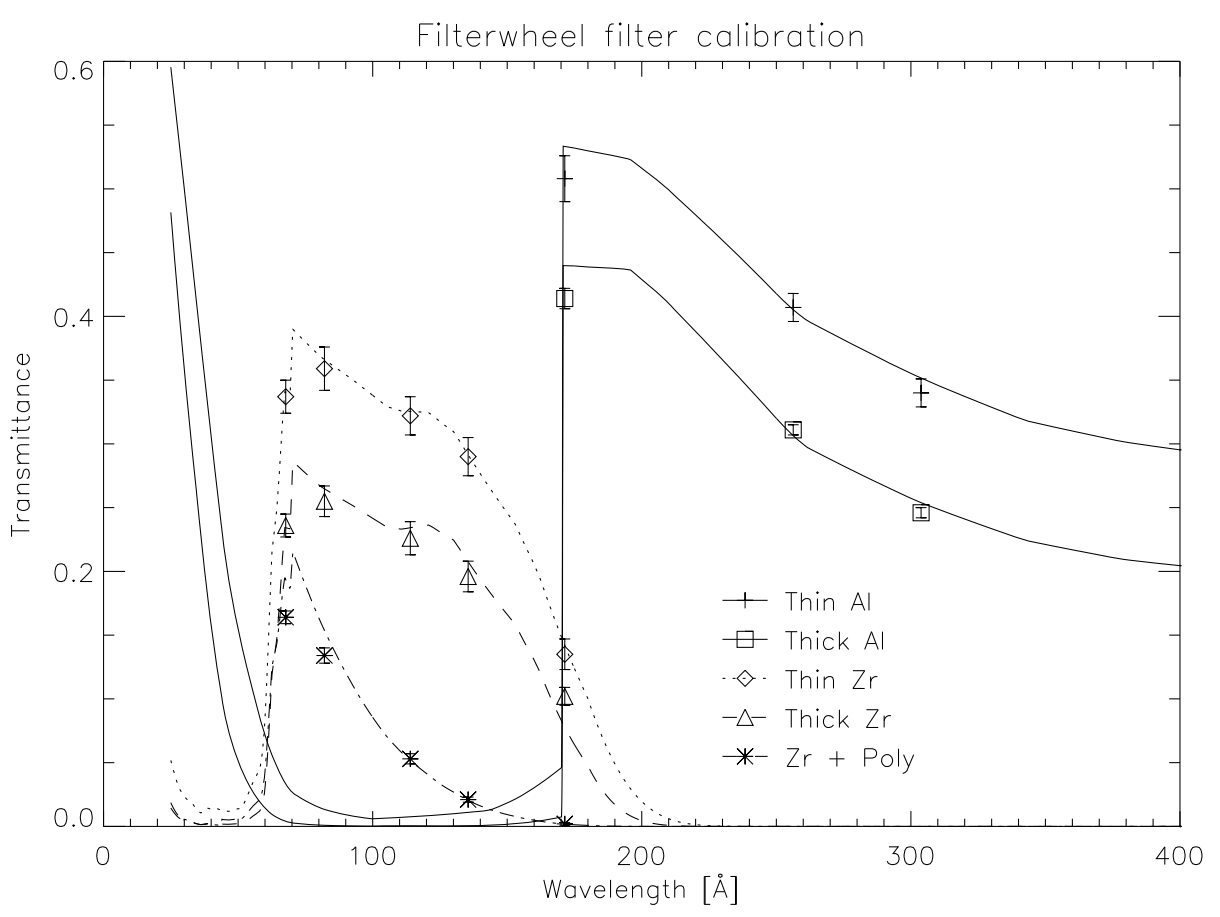

Figure 2 The transmittance of the focal-plane filters, measured at LMSAL, and a model based on optical constants from Henke, Gullikson, and Davis (1993), fit to the measurements. Error bars indicate $1 \sigma$ scatter in a set of measurements made on different filters and coupons.

\subsubsection{Mirrors}

The AIA flight-mirror substrates are made of Zerodur $^{\mathrm{TM}}$ and were figured and polished independently by two different optics vendors: SAGEM (France) and Tinsley (Richmond, California). The high-spatial-frequency roughness of the mirror substrates, which can significantly affect the EUV reflective performance of the multilayer-coated mirrors, was measured by Atomic Force Microscopy at Lawrence Livermore National Laboratory (LLNL); the results are discussed in Soufli et al. (2007). Multilayer coatings are necessary in order to render the mirrors efficient reflectors in the EUV region. The multilayer coating development for the seven EUV AIA channels is discussed in Soufli et al. (2005). Multilayer coatings on the AIA flight-mirror substrates were applied at Reflective X-ray Optics (RXO), LLC for the $94(\mathrm{Mo} / \mathrm{Y}), 131(\mathrm{Mo} / \mathrm{Si}), 304$ and $335(\mathrm{SiC} / \mathrm{Si}) \AA$ channels and at LLNL for the 171,193 and $211(\mathrm{Mo} / \mathrm{Si}) \AA$ channels. The mirrors were then measured $v s$. wavelength across the peak of their response (in-band reflectance measurements) at beamline 6.3.2. of the Advanced Light Source (ALS) synchrotron at Lawrence Berkeley National Laboratory. In-band and off-band reflectance measurements were also performed at the ALS on AIA multilayer witness samples that were deposited at the same time as the flight-mirror coatings in the wavelength region $43-500 \AA$. These measurements were performed three-four years after the flight coatings were deposited, and the results were used in the analysis of the AIA instrument performance, as discussed later in this section. The in-band and off-band AIA measurements on flight mirrors and witnesses will be described in detail in an upcoming 

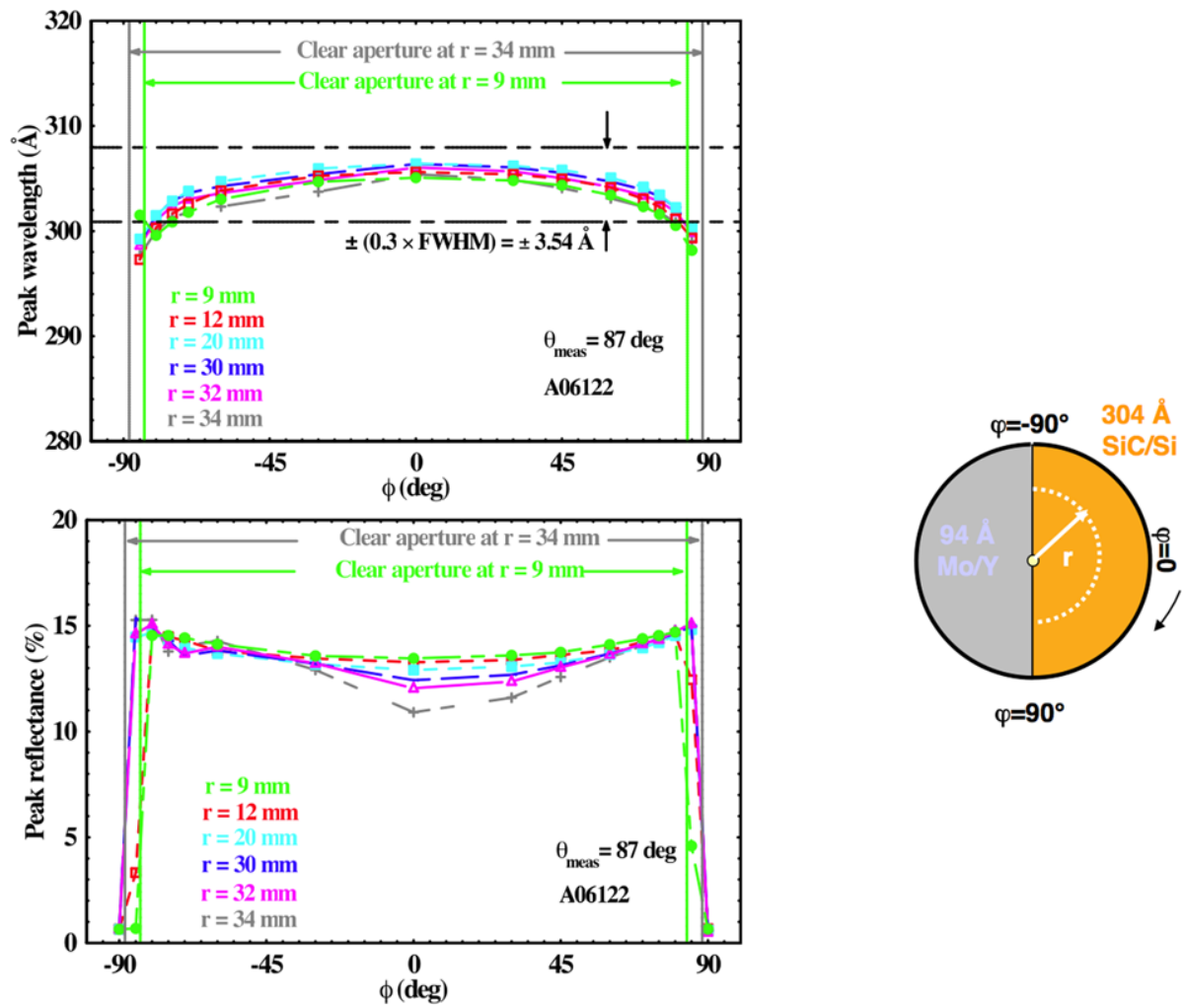

Figure 3 The spatial variation in the peak reflectance and peak wavelength of the SiC-Si multilayer coating on the 304- $\AA$ secondary mirror was sampled in detail, and an area-weighted average was constructed. It should be noted that each azimuth $[\phi]$ scan obtained at radial location $[r]$ corresponds to an area proportional to $r^{2}$, per the drawing on the right.

publication. Two examples from these measurements and analysis are shown in Figures 3 and 4.

For the in-band reflectance measurements, each AIA flight mirror was measured at a number of locations within the clear aperture, as shown in Figure 3. At each location, the reflectance was scanned $v s$. wavelength within the bandpass of each EUV channel. These measurements were combined using an area-weighted average (Podgorski et al., 2009) to produce a single composite reflectivity function for each mirror. There is some variation (of order 10\%) in the measurements taken at different points on the mirror. Note that this standard deviation reflects a real variation in the multilayer across the mirror's surface and is accurately represented by the weighted average of measurements. The individual measurements that go into the mirror calibration are extremely accurate $(<0.6 \%$ error on the measurement of a given point at a given wavelength). There may be some residual error $(<5 \%)$ in the composite reflectivity function due to spatial variations that are not adequately sampled during the mirror calibration. The results of the calibration measurements across a typical mirror are shown in Figure 3.

The other potential error source in the mirror calibration is a change in the mirror performance (due to aging effects such as oxidation of the top layer of the multilayer coating) between the calibration measurement (typically done within a day to roughly one month 


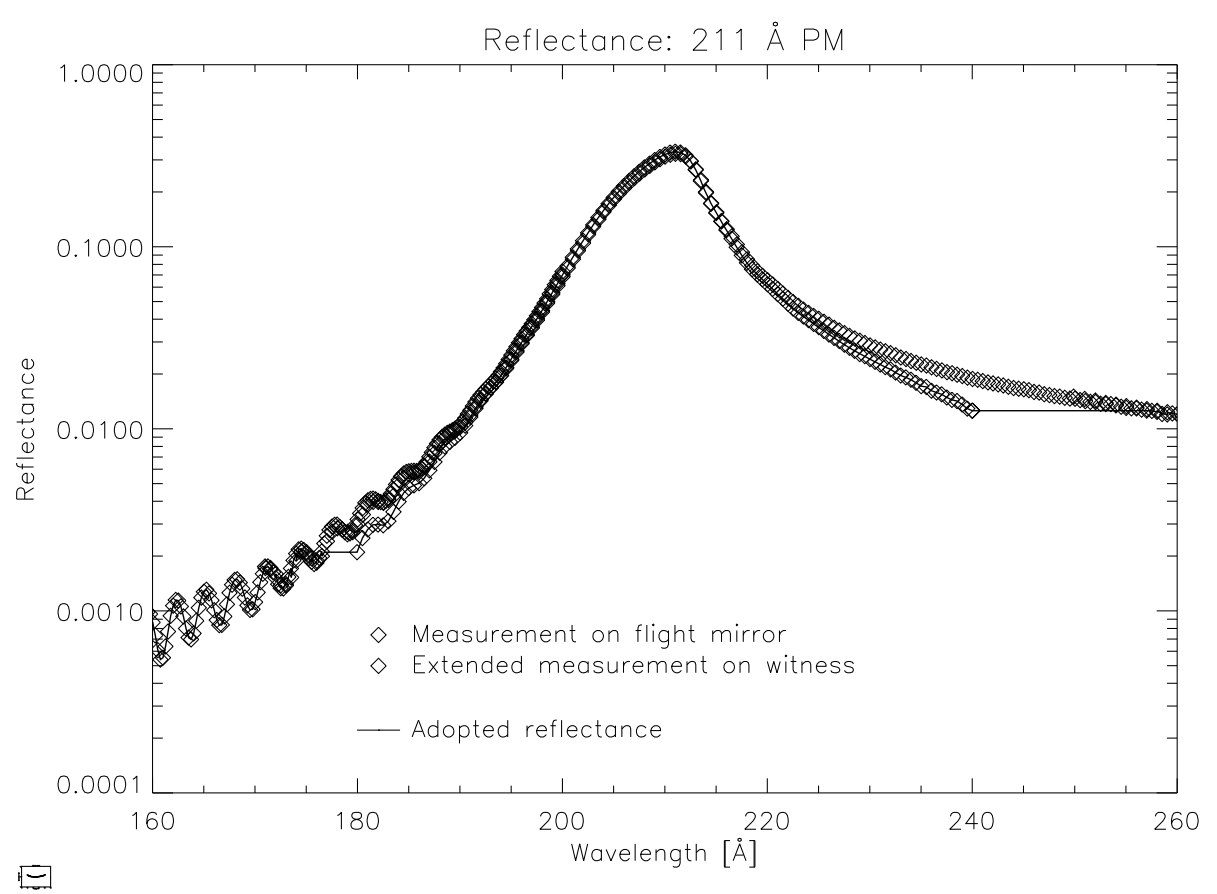

Figure 4 An example of using the full wavelength range measurements from a coating witness to extend the calibration of the flight mirror (in this case, the $211-\AA$ primary).

after the multilayer deposition) and the beginning of on-orbit operations. This change in any case is not expected to exceed 5\%, based on trending measurements of coating-witness samples deposited along with the flight mirrors.

The mirrors were sampled on a very fine wavelength grid, so it is not necessary to fit a model to the measurements in order to interpolate the response in wavelength. However, the original measurements of the mirrors did not extend to wavelengths well away from the reflectance peak. In order to sample the wings of the multilayer reflectance, coating-witness samples that were deposited along with each of the flight-mirror coatings were re-measured at the ALS in 2009 over the wavelength range from $43-500 \AA$. The results were combined with the area-weighted average measurements of the core of the mirror-reflectance curves, as depicted in Figure 4. The flight-mirror measurements were used in the wavelength range where they exist; for extended wavelengths, the calibration adopts the lesser of the nearest flight measurement or the witness measurement. The accuracy of the extended-wavelength measurements on flight witness samples (when applied to the flight mirrors) is not as good as that of the measurements on the flight mirrors themselves, but in general the impact of this portion of the multilayer response (or the portion outside of the range of the witness-sample measurements) on the spectral content of the channel's images is not significant. The one notable exception is the second-order peak of the multilayers for the $335-\AA$ channel, which falls at $183.5 \AA$. This is discussed further in Section 2.2.1.

Figure 5 shows the results of the mirror calibration measurements for the AIA EUV channels. 


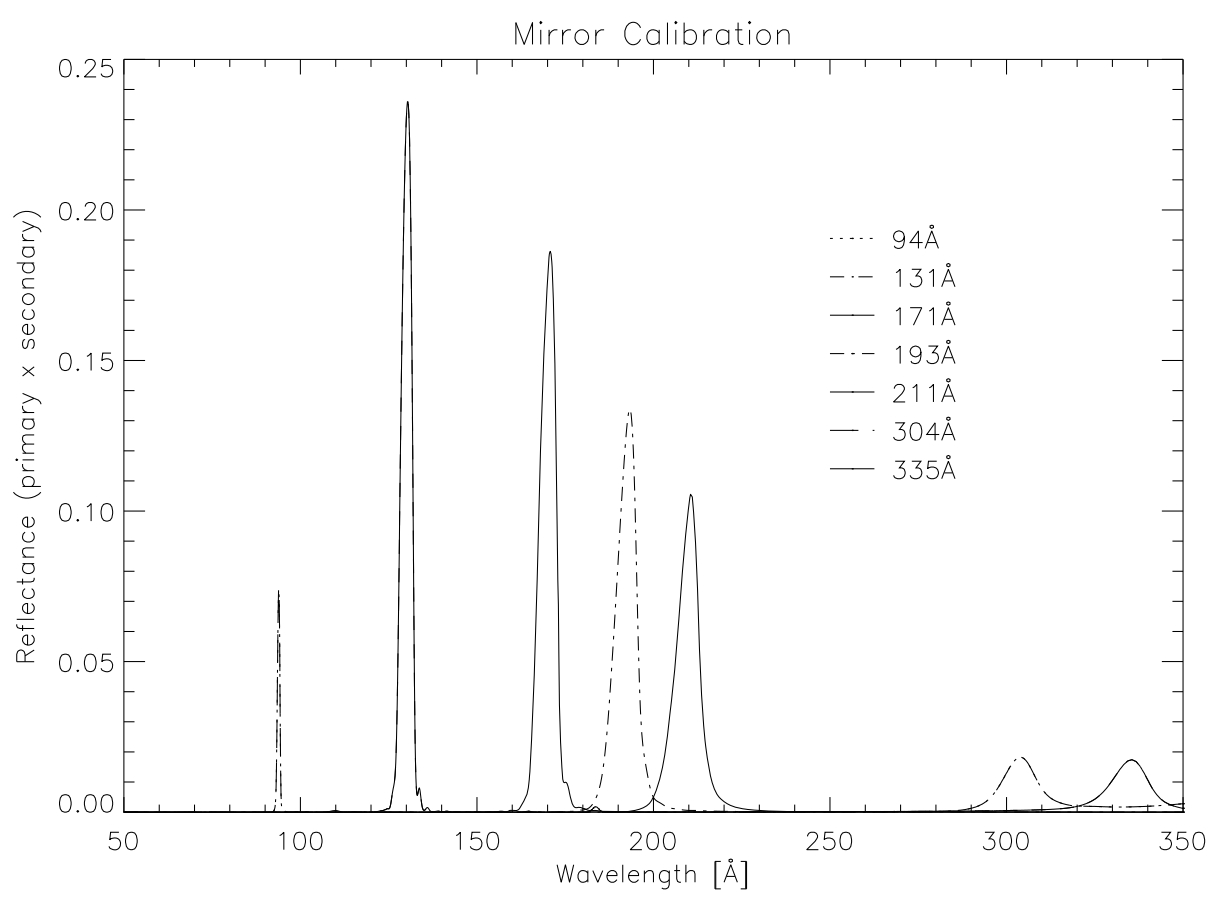

Figure 5 The combined reflectance of the primary and secondary mirrors for the EUV channels.

\subsubsection{CCDs}

Each AIA telescope uses a 16-megapixel back-illuminated, ion-implanted, and laserannealed CCD manufactured by e2v Technologies. The CCDs are divided into four $2048 \times$ 2048 quadrants, which are read out separately and simultaneously at a $2 \mathrm{Mpix} \mathrm{sec}^{-1}$ rate with camera electronics made by the Rutherford Appleton Laboratory (RAL). A discussion of the CCD and camera electronics design and fabrication is given by Waltham et al. (2011). Prior to mounting in the AIA telescopes, the CCDs were screened and calibrated using the LMSAL XUV Calibration facility (Windt and Catura, 1988; Stern et al., 2004) and an ARC Gen II camera system.

Each flight CCD was illuminated in each quadrant at a number of discrete EUV, soft $\mathrm{X}$-ray, and UV wavelengths, and the intensity of the incoming beam was measured using a proportional counter or a microchannel plate (MCP) cross-calibrated with NIST diodes. Unlike the measurements of the filters and mirrors, the CCD calibration measurements rely on the absolute calibration of the reference detector used in the experiment. Variations in the accuracy of the beam normalization due to changes or errors in the calibration of the microchannel plate or proportional counter are the dominant source of uncertainty in the QE measurement $(<5 \%$ for the proportional counter, and around $10 \%$ for the cross-calibrated $\mathrm{MCP}$ ). After accounting for the effect of the CCD-camera system gain (see below), the measurements were used to constrain a model of the detector quantum efficiency (Stern $e t$ al., 1994, 2004). The measurements of the four flight CCDs all agreed to within 5-6\%, less than their error bars, so the mean CCD QEs were fit to the same CCD model, which was used for all channels. The results of the CCD calibration are shown in Figure 6. The model shows excellent agreement with the measurements over the range of the AIA EUV channels 


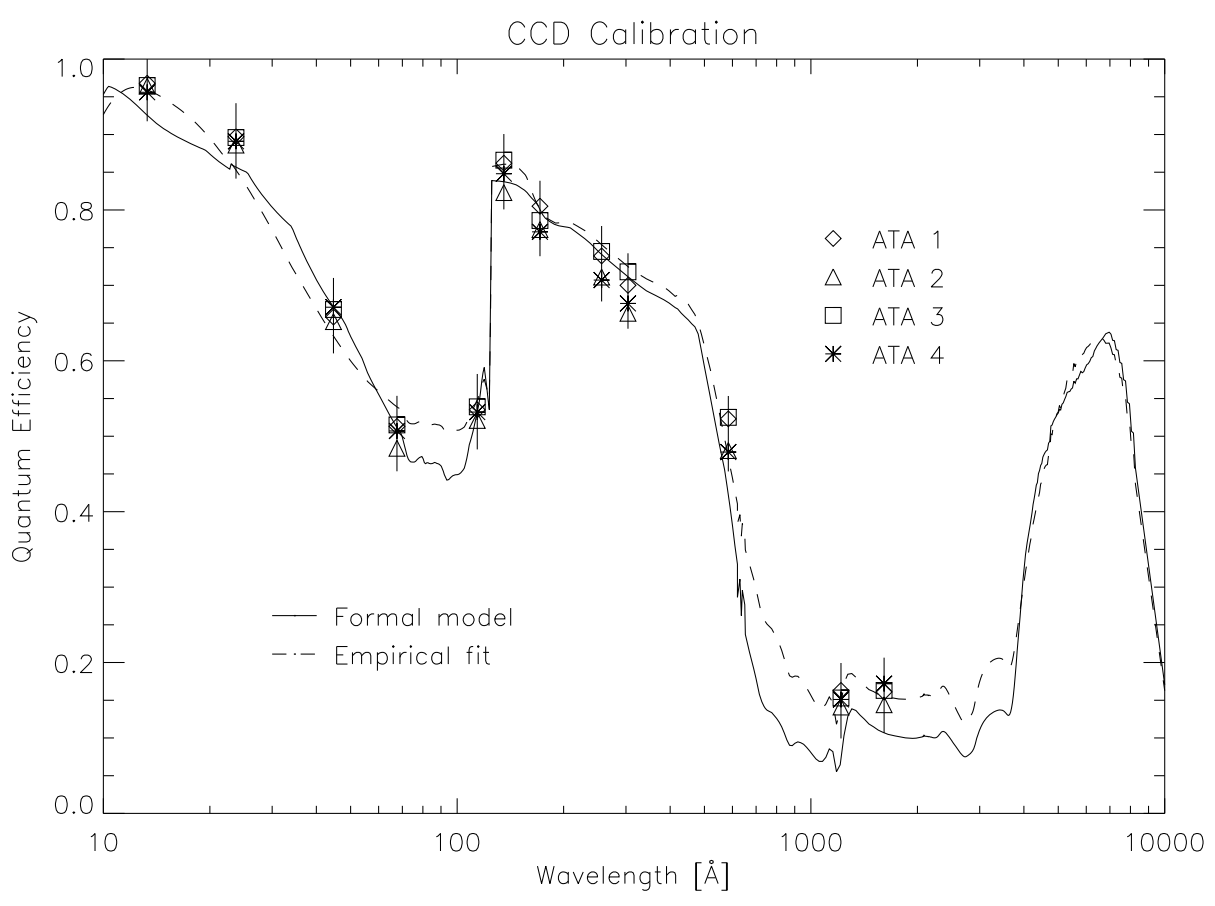

Figure 6 The quantum efficiency of the CCDs measured at LMSAL, and a model fit to the measurements. Agreement between the measurements of the different flight devices and the model is excellent in the EUV range.

and additional data taken in the soft X-ray range; the agreement is not as good for the UV wavelength range. The uncertainties in both the optical constants that go into the model and the QE measurements are larger in the UV range. Therefore, instead of the formal model of the QE that was used for the EUV channels based upon an estimated implant distribution (see Stern, Shing, and Blouke (1994) for the description of the formal and empirical model approaches), we used an empirical fit to the CCD measurements in the calibration of the UV channels. In addition, the CCD response is flat over the bandpass of the AIA UV channels, so the accuracy of the CCD calibration for the UV channels can likely be improved by on-orbit cross-calibration with instruments such as TIMED/SEE (Woods et al., 2005).

\subsubsection{UV Optics}

Like the EUV channels, the UV channel uses half of the aperture of one of the telescopes, but it can record images in three distinct wavelength channels - $1600 \AA, 1700 \AA$, and $4500 \AA$ - using different focal-plane filters. The mirror coatings for the UV channel are Al with a $\mathrm{MgF}_{2}$ overcoating, optimized for reflectance at $1600 \AA$. The mirror coatings were deposited by Acton Research Optics (ARO). The UV channel mirror reflectance was not measured; a model for the UV channel reflectance was calculated using data and code from the internet materials database (IMD: Windt, 1998). The model reflectance agrees well with measurements of similar coatings performed by ARO.

Instead of thin-film filters at the entrance and in the filterwheel, the UV channel uses a $\mathrm{MgF}_{2}$ window with a metal-dielectric bandpass coating to limit visible - IR throughput and 
select a narrow range of UV wavelengths. The $1600-\AA$ channel uses a similar $\mathrm{MgF}_{2}$ optic in the filter wheel to further narrow the bandpass. The 1700- $\AA$ channel has a fused-silica filter with metal-dielectric coatings in the filter wheel, while the 4500 - $\AA$ channel uses a more conventional three-cavity bandpass filter. The UV window and focal-plane filters were made by ARO; the $4500 \AA$ focal-plane filter was made by Andover Corporation. While the UV window and $4500 \AA$ focal-plane filter are plano-plano optics, the $1600-\AA$ and $1700-\AA$ focal-plane filters each has a convex surface in order to keep them parfocal with the $171-\AA$ channel that shares their telescope assembly.

The UV window and filters were calibrated using an ARO FUV spectrophotometer. A Cary spectrophotometer was used to measure the out-of-band (NUV-visible) transmittance.

\subsubsection{Contamination}

AIA was kept in a clean environment throughout integration and test; cleanliness was strictly maintained even during spacecraft-level integration due to the sensitivity of both AIA and SDO's Extreme Ultraviolet Variability Experiment (EVE) to molecular contamination. Nevertheless, it is inevitable that some amount of contamination will accumulate on the optics and (especially) on the detector during instrument operations, and our throughput model reflects this.

A venting analysis of the telescope system suggested that a total effective path length of a few hundred Ångstroms of volatile molecules would likely accumulate in the AIA optical system. We used IMD to model the EUV throughput of a typical contaminant, Triphenyl Phosphate $\left(\mathrm{C}_{18} \mathrm{H}_{15} \mathrm{O}_{4} \mathrm{P}\right)$, at a thickness of $275 \AA$, and included this in our preflight estimate of the system throughput. The contaminant transmittance curve is shown in Figure 7. Note that it has the strongest effect in the longer-wavelength channels, especially $304 \AA$ and $335 \AA$ A.

There were no preflight measurements that could constrain the thickness of the contaminant layer, so the uncertainty on the contamination function is as large as the correction itself. We expect to adjust this parameter in order to achieve better agreement with reference spectra from EVE and other instruments during on-orbit cross-calibration, and may use the thickness of the contaminant layer as a parameter to optimize the ongoing cross-calibration.

The throughput of contaminants at UV and visible wavelengths is more difficult to model analytically; therefore we did not include an allocation for contamination in the initial calibration functions for the UV channels. Corrections for the effect of contamination will be done empirically by cross-calibration with other instruments.

\subsection{Wavelength Response}

Table 2 illustrates how the end-to-end wavelength-response functions $[R(\lambda)]$ are calculated from the component-level measurements by showing the efficiency of each component and the total instrument response at the wavelength of the strongest solar emission line in each channel. The effective areas for the seven EUV channels as a function of the wavelength are plotted in Figure 8. The effective areas for the three UV-Vis channels as a function of the wavelength are shown in Figure 9.

\subsubsection{Channel Crosstalk}

For three of the four AIA telescopes, both channels (both halves of the aperture) are illuminated at all times, and the active channel is selected using the focal-plane filters. (The 


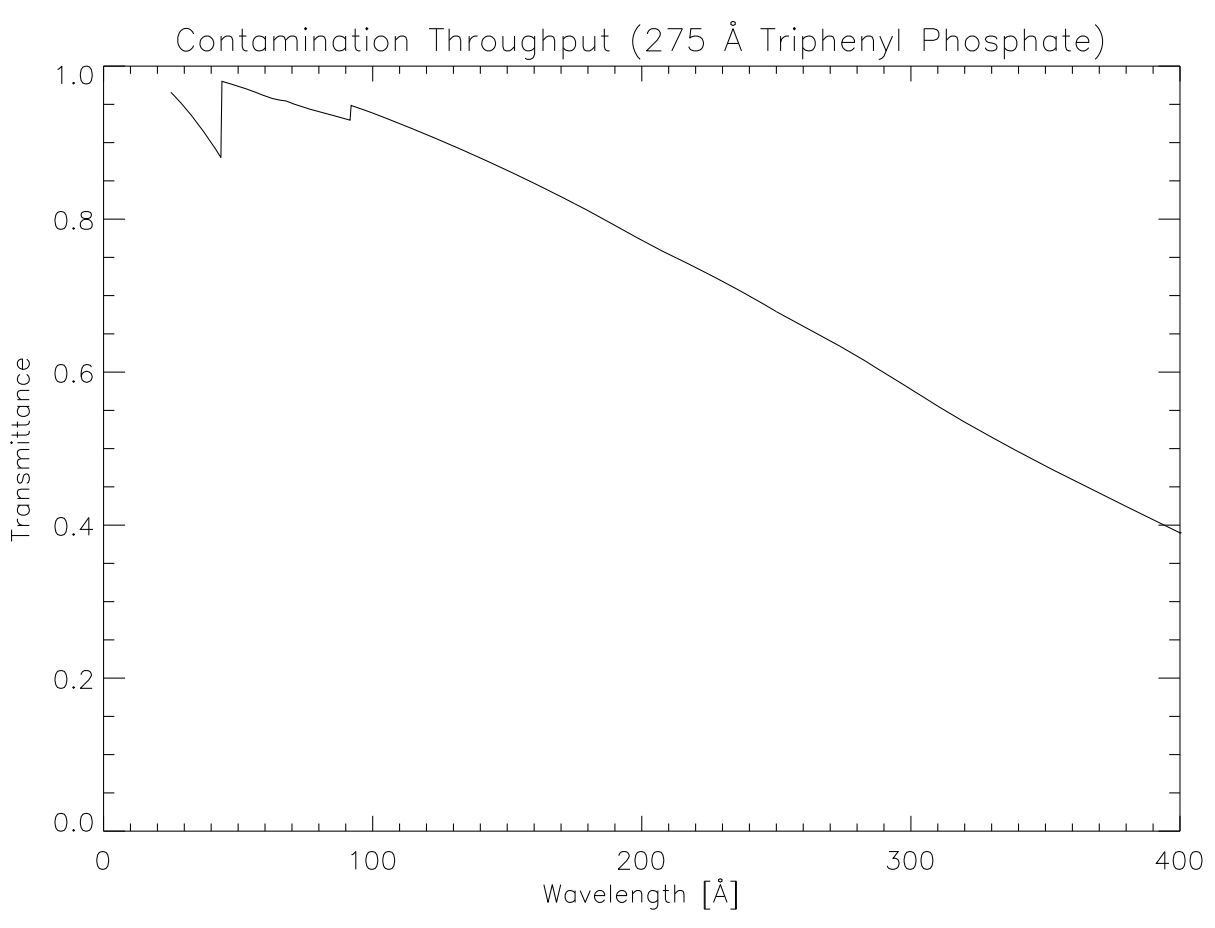

Figure 7 Transmittance for the model contamination layer assumed in the preflight calibration.

exception is telescope 2, which includes the 193- $\AA$ and 211- $\AA$ channels, where a mechanical aperture selector is used to block half of the aperture in order to select the active channel for an image.) While the wavelength channels were grouped to minimize crosstalk, the focalplane filters on telescopes 1 (which includes the 131- and 335- $\AA$ channels) and 4 (94 and $304 \AA$ ) do not perfectly reject light from the opposite channel, so there is some crosstalk between the wavelength channels.

This is most significant for the $335-\AA$ channel: it has a relatively low reflectivity, and is paired with the highly reflective $131-\AA$ channel. The effective area of the $335-\AA$ channel (using thin and thick focal-plane filters) is shown over a wide wavelength range in Figure 10. The contribution of the 131- $\AA$ coatings on the opposite half of the telescope aperture, as well as the higher-order peak from the 335 - $\AA$ multilayer mirror discussed above, are evident. We have estimated the relative contribution of the $131-\AA$ coatings to the 335 - $\AA$ channel images by folding solar spectra through the effective-area function. This analysis demonstrates that, while the $131-\AA$ channel generally accounts for less than $2 \%$ of the total counts in the $335-\AA$ images in quiet Sun or active regions, it can contribute up to $40 \%$ during flares (when the hot lines around $131 \AA$ are enhanced substantially compared to the 335 - $\AA$ Fe XVI line). The crosstalk can be minimized (at the expense of throughput) by using the thick focal-plane filter on that channel. However, given the low level of solar activity thus far in this solar cycle, we have continued to primarily use the thin filter.

\subsection{Error Budget}

An estimate of the error in the photometric calibration can be calculated by adding the fractional errors on each component-level calibration in quadrature (see Table 3 ). The calibration 
Table 2 The components of the wavelength-response function at the strongest line in each channel.

\begin{tabular}{llllllll}
\hline \multirow{2}{*}{ Component } & \multicolumn{7}{l}{ Wavelength of strongest line $[\AA]$} \\
\cline { 2 - 7 } & 93.9 & 131.2 & 171.1 & 195.1 & 211.3 & 303.8 & 335.4 \\
\hline$A_{\text {geo }}\left[\mathrm{cm}^{2}\right]$ & & & 83.0 & & & \\
$T_{\mathrm{E}}$ & 0.348 & 0.306 & 0.533 & 0.523 & 0.497 & 0.352 & 0.324 \\
$R_{\mathrm{P}}$ & 0.241 & 0.505 & 0.424 & 0.283 & 0.331 & 0.117 & 0.117 \\
$R_{\mathrm{S}}$ & 0.308 & 0.399 & 0.434 & 0.303 & 0.305 & 0.129 & 0.125 \\
$T_{\mathrm{F}}$ & 0.348 & 0.306 & 0.533 & 0.523 & 0.497 & 0.352 & 0.324 \\
$Q$ & 0.442 & 0.838 & 0.801 & 0.779 & 0.774 & 0.712 & 0.696 \\
$D$ & 0.946 & 0.893 & 0.827 & 0.782 & 0.752 & 0.569 & 0.504 \\
$A_{\mathrm{eff}}$ & 0.312 & 1.172 & 2.881 & 1.188 & 1.206 & 0.063 & 0.045 \\
$G\left[\mathrm{DN} \mathrm{phot}{ }^{-1}\right]$ & 2.128 & 1.523 & 1.168 & 1.024 & 0.946 & 0.658 & 0.596 \\
$R(\lambda)\left[\mathrm{cm}^{2} \mathrm{DN} \mathrm{phot}^{-1}\right]$ & 0.664 & 1.785 & 3.365 & 1.217 & 1.14 & 0.041 & 0.027 \\
\hline
\end{tabular}

\begin{tabular}{llll}
\hline Component & \multicolumn{3}{l}{ Nominal bandpass center $[\AA]$} \\
\cline { 2 - 4 } & 1600 & 1700 & 4500 \\
\hline$A_{\text {geo }}\left[\mathrm{cm}^{2}\right]$ & & 30.8 & \\
$T_{\mathrm{E}}$ & 0.114 & 0.053 & $3.80 \times 10^{-5}$ \\
$R_{\mathrm{P}}$ & 0.9 & 0.904 & 0.884 \\
$R_{\mathrm{S}}$ & 0.9 & 0.904 & 0.884 \\
$T_{\mathrm{F}}$ & 0.043 & 0.191 & 0.705 \\
$Q$ & 0.155 & 0.153 & 0.442 \\
$A_{\text {eff }}$ & $1.92 \times 10^{-2}$ & $3.89 \times 10^{-2}$ & $2.83 \times 10^{-4}$ \\
$G\left[\mathrm{DN} \mathrm{phot}{ }^{-1}\right]$ & 0.125 & 0.118 & 0.044 \\
$R(\lambda)\left[\mathrm{cm}^{2} \mathrm{DN} \mathrm{phot}^{-1}\right]$ & $2.4 \times 10^{-3}$ & $4.6 \times 10^{-3}$ & $1.3 \times 10^{-5}$ \\
\hline
\end{tabular}

error for each component is the estimated $1 \sigma$ difference between the value of the component efficiency that has been adopted into the calibration model at a given wavelength and the true efficiency of the component at that wavelength. (Note that the errors in Table 3 are approximate, and do not include subtleties such as the fact that certain errors affect some channels or some wavelengths more than others.) The largest terms are the uncertainty in the contamination thickness and the CCD-QE determination. The overall accuracy of the preflight calibration is estimated to be of order $25 \%$.

\subsection{Cross-Calibration with EVE}

EVE (Woods et al., 2010) measures the solar spectral irradiance at $1 \AA$ spectral resolution over much more than the full wavelength range of AIA's EUV channels, and at a very similar cadence to AIA. EVE expects to maintain an absolute calibration accuracy $\approx 20 \%$ by cross-calibrating with a series of sounding-rocket underflights; the first rocket underflight went off successfully on 3 May 2010. AIA will take advantage of EVE's excellent absolute calibration to refine our estimate of the instrument-response functions.

A rather crude cross-calibration can be obtained by integrating the EVE irradiance measurements $\left[I(\lambda)\right.$ : phot $\left.\mathrm{cm}^{-2} \mathrm{~s}^{-1} \AA^{-1}\right]$ times the AIA response functions 

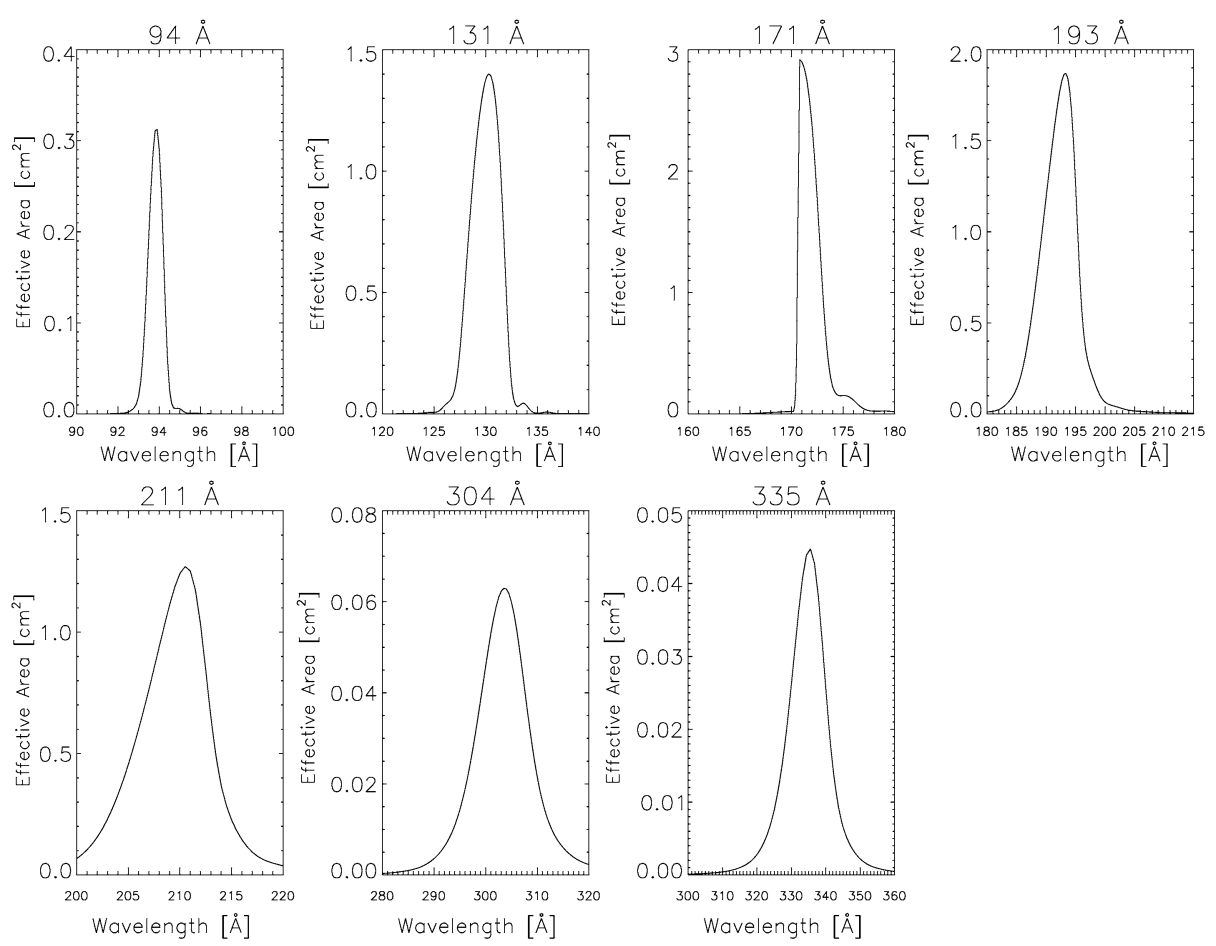

Figure 8 EUV effective-area functions.

Table 3 Calibration error budget. Sources of calibration are (M)odel, experimental (D)ata, or a combination of the two where data at selected wavelengths are used to constrain a model (DM).

\begin{tabular}{llll}
\hline Component & Calibration Error [\%] & Source of Calibration & Dominant Source of Error \\
\hline$T_{\mathrm{E}}$ & 7 & $\mathrm{M}$ & $\begin{array}{l}\text { Uncertainty in optical constants; } \\
\text { differences between measured filter } \\
\text { samples and flight filters }\end{array}$ \\
& & & $\begin{array}{l}\text { Degradation in mirror after calibration } \\
R_{\mathrm{P}}\end{array}$ \\
$R_{\mathrm{S}}$ & 6 & $\mathrm{D}$ & $\begin{array}{l}\text { Degradation in mirror after calibration } \\
T_{\mathrm{F}}\end{array}$ \\
$D$ & 5 & $\mathrm{D}$ & Uncertainty in optical constants \\
$Q$ & 20 & $\mathrm{DM}$ & Uncertainty in contaminant thickness \\
& 15 & $\mathrm{M}$ & $\begin{array}{l}\text { Absolute calibration of reference } \\
\text { detector; uncertainty in optical constants }\end{array}$ \\
Total & 28 & $\mathrm{DM}$ & \\
\hline
\end{tabular}

$\left[R(\lambda): \mathrm{cm}^{2} \mathrm{DN}_{\text {phot }}{ }^{-1}\right]$ to predict the total count rate that AIA should observe (in DN sec${ }^{-1}$ ), integrated over the full image, and then comparing this prediction with the actual full-frame count rate. This approach is limited in a number of ways - it ignores the fact that AIA's field of view is somewhat smaller than EVE's, and it is subject to errors up to $40 \%$ due to the width of EVE's spectral response (particularly for AIA channels with relatively sharp fea- 


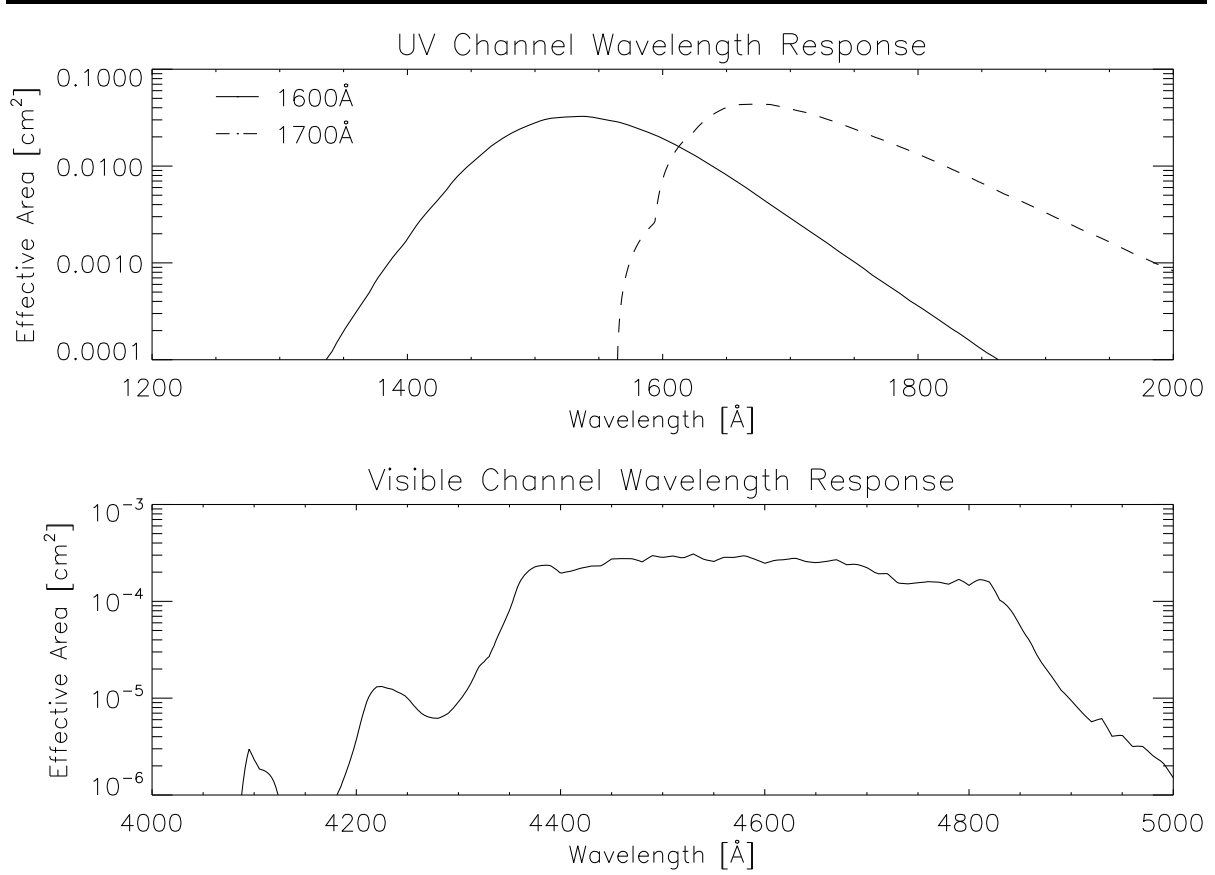

Figure 9 UV-Vis channel effective-area functions.

Table 4 Cross-calibration between the AIA EUV channels and the EVE calibration rocket underflight on 3 May 2010; and between the AIA UV channels and TIMED/SEE observations on the same date.

\begin{tabular}{llll}
\hline Channel & \multicolumn{2}{l}{ Full-disk count rate $\left[\mathrm{DN} \mathrm{s}^{-1}\right]$} & $\begin{array}{l}\text { Ratio } \\
\text { predicted/observed }\end{array}$ \\
\cline { 2 - 3 } & $\begin{array}{l}\text { Predicted from EVE } \\
\text { rocket data }\end{array}$ & $\begin{array}{l}\text { Observed by } \\
\text { AIA }\end{array}$ & \\
\hline $94 \AA$ & $3.3 \times 10^{7}$ & $3.6 \times 10^{7}$ & 0.90 \\
$131 \AA$ & $8.3 \times 10^{7}$ & $7.3 \times 10^{7}$ & 1.15 \\
$171 \AA$ & $1.7 \times 10^{9}$ & $1.8 \times 10^{9}$ & 0.91 \\
$193 \AA$ & $1.7 \times 10^{9}$ & $1.9 \times 10^{9}$ & 0.91 \\
$211 \AA$ & $5.6 \times 10^{8}$ & $5.6 \times 10^{8}$ & 1.00 \\
$304 \AA$ & $3.0 \times 10^{8}$ & $4.4 \times 10^{8}$ & 0.69 \\
$335 \AA$ & $4.3 \times 10^{7}$ & $5.9 \times 10^{7}$ & 0.73 \\
\hline
\end{tabular}

\begin{tabular}{llll}
\hline \multirow{2}{*}{ Channel } & \multicolumn{2}{l}{ Full-disk count rate $\left[\mathrm{DN} \mathrm{s}^{-1}\right]$} & \multirow{2}{*}{$\begin{array}{l}\text { Ratio } \\
\text { predicted/observed }\end{array}$} \\
\cline { 2 - 3 } & $\begin{array}{l}\text { Predicted from } \\
\text { TIMED/SEE }\end{array}$ & $\begin{array}{l}\text { Observed by } \\
\text { AIA }\end{array}$ & \\
\hline $1600 \AA$ & $1.2 \times 10^{9}$ & $5.6 \times 10^{8}$ & 2.1 \\
$1700 \AA$ & $5.1 \times 10^{9}$ & $6.8 \times 10^{9}$ & 0.75 \\
\hline
\end{tabular}

tures in wavelength, such as the 171- $\AA$ edge or the $94-\AA$ channel). However, as a first-order check this approach has much merit.

This cross-calibration was performed using sample data from the EVE calibration rocket flown on 3 May and AIA images taken at the same time. The results are shown in Table 4. 


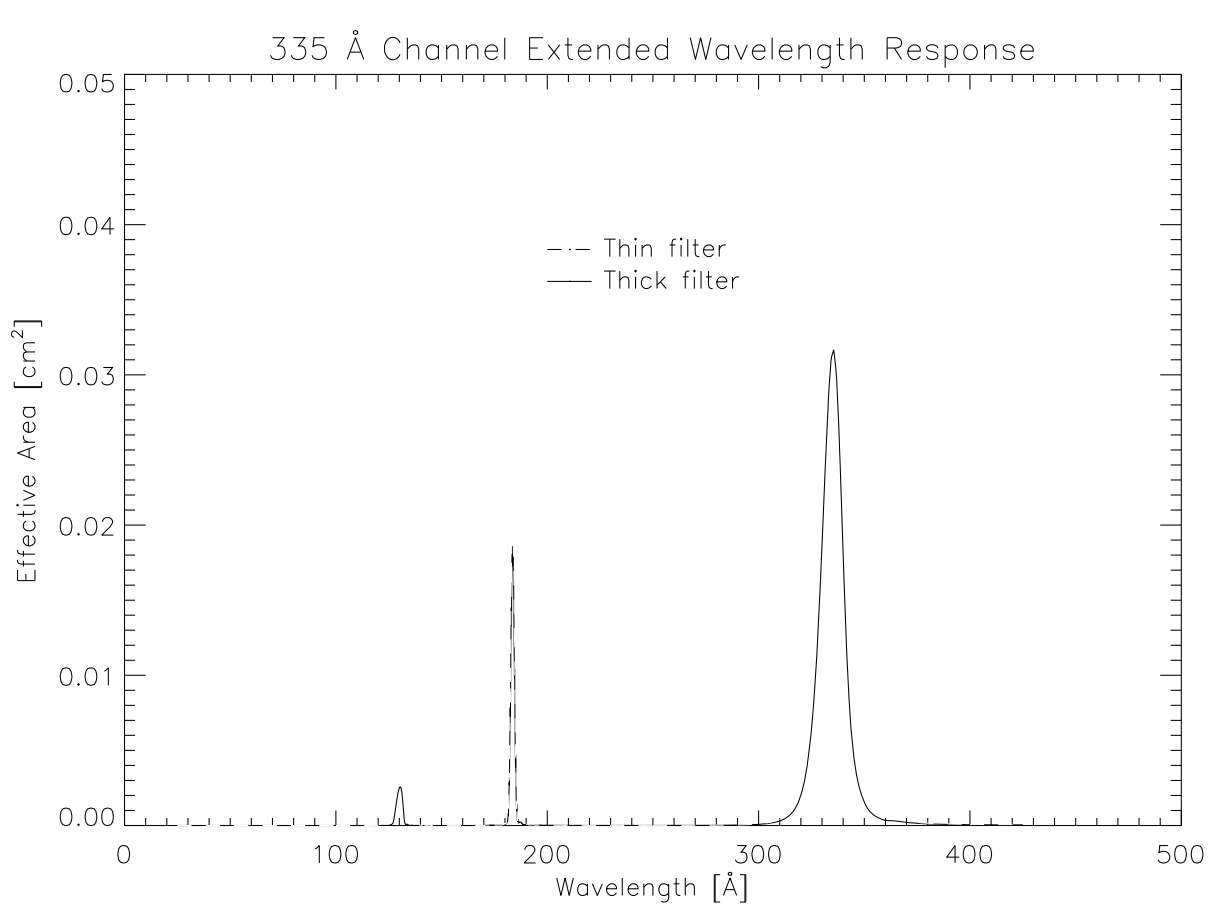

Figure 10 Full wavelength range effective area for the 335 - $\AA$ channel, showing the effects of crosstalk from the 131- $\AA$ channel which shares the aperture of telescope 1 , and a second-order peak in the mirror reflectivity around $184 \AA$. The other EUV channels do not show any significant throughput outside of the range shown in Figure 8.

The agreement is good, and likely can be improved by some straightforward updates to the AIA response functions (such as optimizing the thickness of the contaminant layer, which may be throwing off the predictions for the $304-\AA$ and $335-\AA$ channels).

Cross-calibration with EVE will be performed routinely during the AIA mission. This will allow us to track any degradation in the instrument. Furthermore, by observing how the ratio of observed AIA intensities to those intensities predicted by combining the EVE measurements and the AIA response functions varies with solar activity, we can refine the shape of the AIA wavelength response and improve the accuracy of the temperature-response function.

\subsection{Temperature Response}

The goal of the instrument calibration is to enable the user to extract useful scientific information from the AIA data. In Equation (1), if all of the effects that go into $p$ and $\eta$ are well known, then the user can obtain information about the emitted solar intensity $[I]$. One way to translate the information about $I$ into information about the thermodynamic state of the corona is to express the solar spectral radiance as an integral over temperature:

$$
I(\lambda, \mathbf{x})=\int_{0}^{\infty} G(\lambda, T) \operatorname{DEM}(T, \mathbf{x}) \mathrm{d} T .
$$

Here $G$ is the contribution function, which contains information on the plasma and atomic physics governing how material at a given temperature emits radiation. Given a few assump- 
Table 5 Count rates predicted using CHIANTI, and observed quiet-Sun count rates.

\begin{tabular}{|c|c|c|c|c|c|}
\hline \multirow[t]{2}{*}{ Channel } & \multicolumn{4}{|c|}{ Predicted count rate [DN pix $\left.{ }^{-1} \mathrm{~s}^{-1}\right]$} & \multirow{2}{*}{$\begin{array}{l}\text { Observed quiet-Sun } \\
\text { count rate } \\
{\left[\mathrm{DN} \text { pix }^{-1} \mathrm{~s}^{-1}\right]}\end{array}$} \\
\hline & Coronal Hole & Quiet Sun & Active Region & Flare & \\
\hline $94 \AA$ & 0.0 & 0.3 & 14.5 & 49249.6 & 0.2 \\
\hline $131 \AA$ & 2.1 & 4.6 & 39.9 & 368727.1 & 4.3 \\
\hline $171 \AA$ & 65.5 & 272.8 & 2711.2 & 63727.1 & 323.9 \\
\hline $193 \AA$ & 9.3 & 106.8 & 1988.8 & 475965.5 & 170.5 \\
\hline $211 \AA$ & 1.7 & 28.3 & 750.5 & 32006.3 & 38.5 \\
\hline $304 \AA$ & 17.4 & 25.5 & 126.8 & 98966.5 & 125.5 \\
\hline $335 \AA$ & 0.3 & 1.4 & 44.6 & 18521.5 & 4.2 \\
\hline
\end{tabular}

tions, it can be calculated using CHIANTI (Dere et al., 1997, 2009). DEM is the differential emission measure function,

$$
\operatorname{DEM}(T)=n_{\mathrm{e}}^{2} \frac{\mathrm{d} z}{\mathrm{~d} T},
$$

which describes the thermal structure of the coronal plasma along the line of sight to the pixel.

It is possible to calculate temperature-response functions $[K(T)]$ for the AIA channels by folding the CHIANTI data and the instrument-wavelength response together:

$$
K_{i}(T)=\int_{0}^{\infty} G(\lambda, T) R_{i}(\lambda) \mathrm{d} \lambda
$$

Then the observations can be expressed as an integral over temperature instead of wavelength:

$$
p_{i}(\mathbf{x})=\int_{0}^{\infty} K_{i}(T) \operatorname{DEM}(T, \mathbf{x}) \mathrm{d} T
$$

and this equation can (in principle) be inverted to obtain a DEM from a given set of observations $[p]$ and an instrument calibration $[K]$.

While the challenges of this problem are discussed in detail in the astrophysical literature (e.g., Pottasch, 1963; Withbroe, 1975; Sylwester, Schrijver, and Mewe, 1980; Judge, Hubeny, and Brown, 1997), it remains a useful approach, as the calculation of the instrument temperature-response functions provides some basic insight into the interpretation of the images from the various channels.

We used CHIANTI to produce a typical spectral contribution function $[G]$. We assumed the coronal abundances of Feldman and Widing (1993), and the ionization balance of Dere et al. (2009) with a constant pressure of $10^{15} \mathrm{~cm}^{-3} \mathrm{~K}$. With this contribution function, we computed temperature-response functions for the AIA EUV channels. The results are shown in Figure 11. We then combined these temperature-response functions with the DEM functions distributed with CHIANTI to predict count rates for typical solar features. The results are shown in Table 5, along with observed count rates from a patch of quiet Sun. Note that we have included an empirical correction factor of $20 \times$ on the He II $304-\AA$ intensities. The He II line at $304 \AA$ is not well-modeled by CHIANTI, and generally should not be used in DEM analysis (Warren, 2005). 


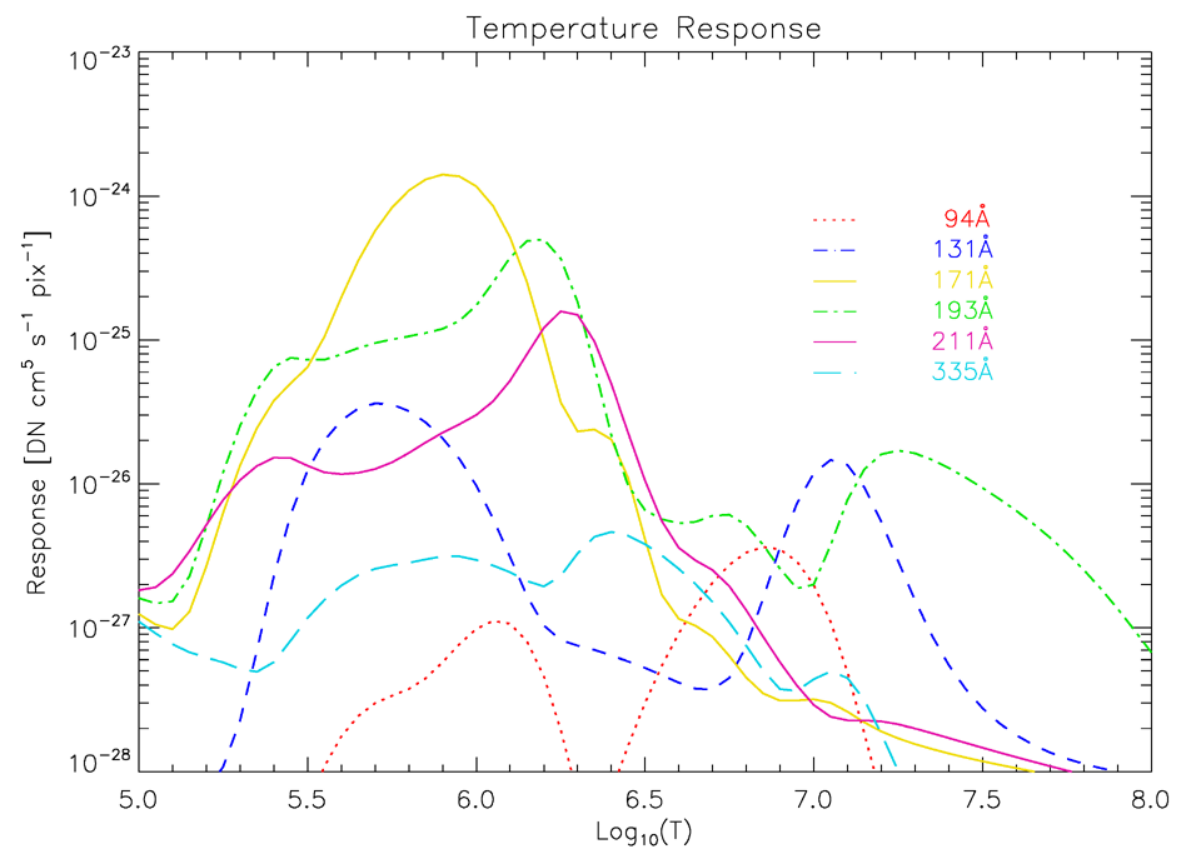

$94 \AA$

$131 \AA$

$171 \AA$
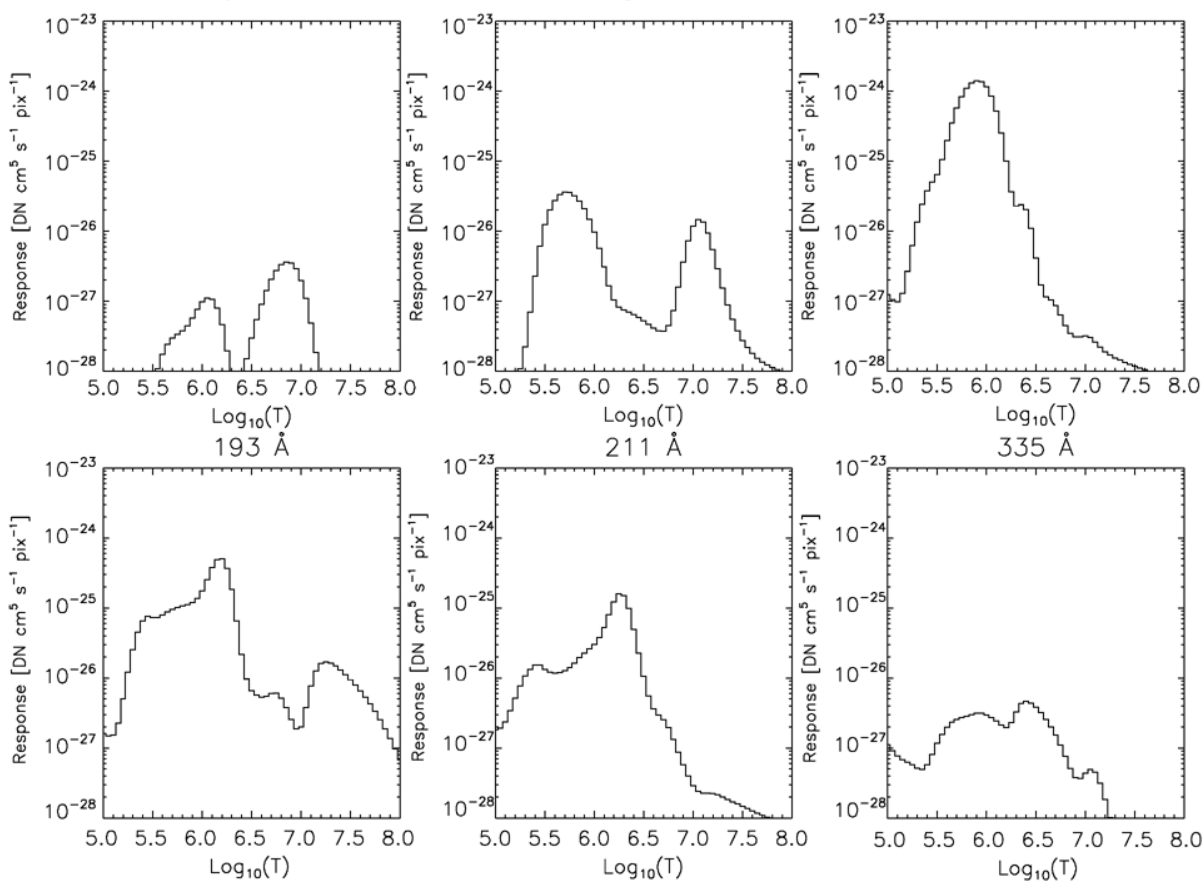

Figure 11 Temperature-response functions for the six EUV channels dominated by Fe emission, calculated from the effective-area functions and a CHIANTI model of solar emissivity. 


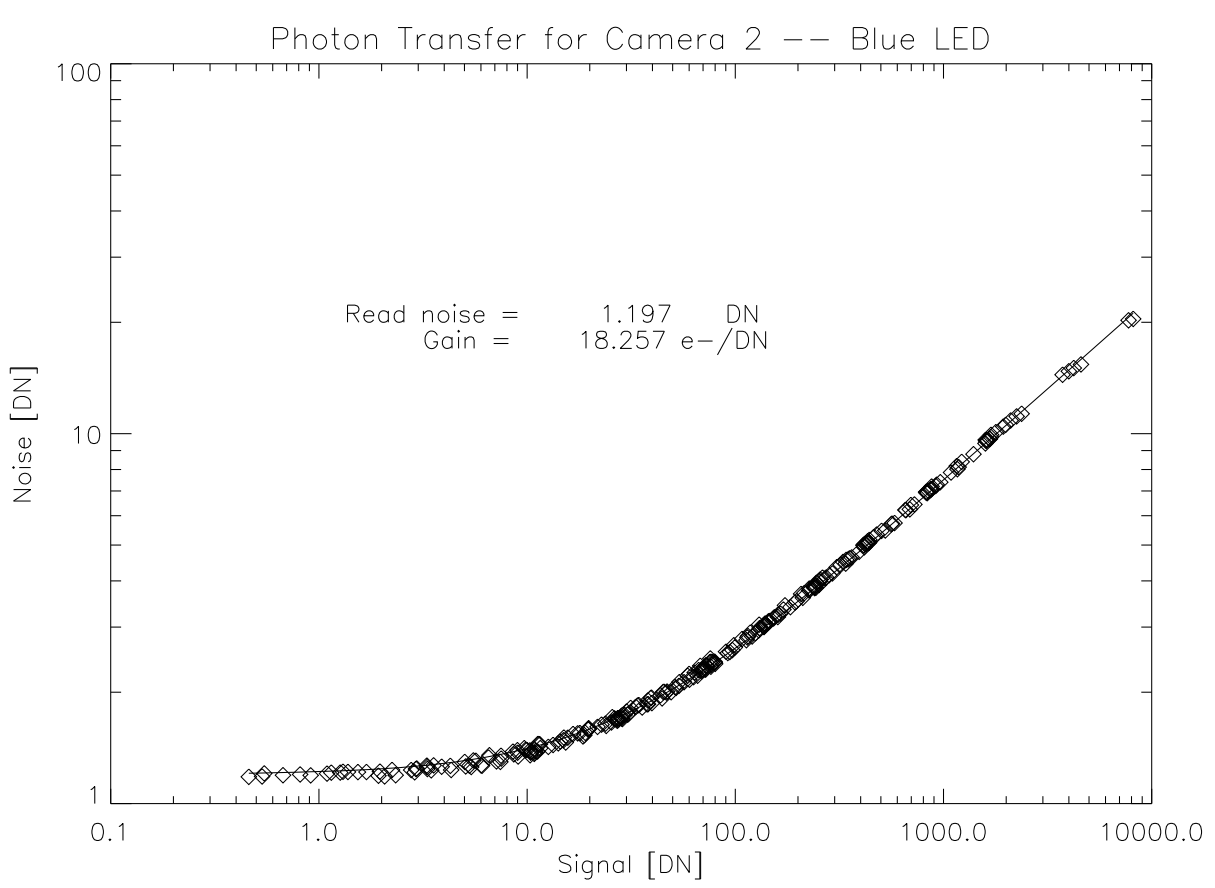

Figure 12 A sample photon-transfer curve for the CCD-camera system on ATA2. The slope of the linear part of the curve gives the gain of the system in electrons per DN; this gain is incorporated into the wavelength-response functions. The level at which the curve flattens out gives the read noise.

The default temperature-response and wavelength-response functions can be accessed through the SolarSoft (SSW) IDL library (Freeland and Handy, 1998), as described in the Appendix.

\section{Instrument Characterization}

\subsection{Camera-CCD System Characterization}

The AIA cameras use a 14-bit analog-to-digital converter (ADC) to translate the charge read out from each pixel to a digital number [DN] value. The gain of the camera (in electrons $\mathrm{DN}^{-1}$ ) directly affects the accuracy of the instrument calibration. The camera gain was measured prior to launch both using ${ }^{55} \mathrm{Fe} \mathrm{X}$-rays and at visible wavelengths. In visible light, the gain is determined by measuring the camera photon-transfer curve. A dark image is taken along with a pair of light images (to eliminate pixel-to-pixel variation), and for a number of subregions within the images the difference between the light images is plotted $v s$. their average value. This is done for a series of increasing integration times, producing a curve like the one in Figure 12. For large-enough signals, the difference between the images is proportional to the shot noise (the square root of the number of photons), so the number of photons can be determined for a given DN value (and thus the number of detected electrons, and the number of electrons $\mathrm{DN}^{-1}$ ). For small signals, the difference goes to a constant value, which is the read noise of the camera system in DN. Note that, in all cases, the read noise is $\approx 20-22 \mathrm{e}^{-} \mathrm{RMS}$, corresponding to, e.g., the charge produced and collected by fewer than 
Table 6 Camera system gain and read noise.

\begin{tabular}{llll}
\hline Camera & $\begin{array}{l}\text { Wavelengths } \\
{[\AA]}\end{array}$ & $\begin{array}{l}\text { Gain } \\
{\left[\mathrm{e}^{-} \mathrm{DN}^{-1}\right]}\end{array}$ & $\begin{array}{l}\text { Read Noise } \\
{[\mathrm{DN}]}\end{array}$ \\
\hline 1 & 131,335 & 17.6 & 1.18 \\
2 & 193,211 & 18.3 & 1.20 \\
3 & $171, \mathrm{UV}-$ Vis & 17.7 & 1.15 \\
4 & 94,304 & 18.3 & 1.14 \\
\hline
\end{tabular}

three photons at $304 \AA$. Thus, for all practical purposes, the images are photon-noise limited except at the faintest signal levels. The gains of the four AIA cameras are listed in Table 6.

The camera-CCD system gain is a weak function of temperature, and may change as the camera electronics age. Therefore, the light-transfer curve will be checked periodically on orbit using solar EUV illumination and visible light from the blue LED built into the telescope spider assemblies.

The light-transfer curve also provides information about the linearity of the camera-CCD system (from the ratio of average signal to exposure time). The AIA camera system is linear to better than $1 \%$ for signals up to $11000 \mathrm{DN}$.

\subsection{Flat-Field}

The instrument flat-field has been measured on orbit using a variety of techniques. Most of the data were taken by sending a series of small offsets to the active secondary mirror, displacing the image to $\approx 12$ different positions with shifts of up to $\approx 35$ arcsecs. Larger scale offsets were obtained during a coordinated spacecraft maneuver that sent SDO to a pattern of offpoints with a scale of about 25 arcmins. The offpoint data were analyzed using a Kuhn algorithm (Kuhn, Lin, and Loranz, 1991) to produce a map of the sensitivity of different parts of the image. The flat-field is normalized to a mean value of one.

A sample flat-field for the 171- $\AA$ channel is shown in Figure 13. It consists of several distinctly recognizable components: the quadrant scaling due to slight gain mismatches between the four amplifiers used to read out each CCD; a "tire track" pattern that consists of diagonal stripes with a variation of a few percent, characteristic of the CCD laser-anneal process; the grid of the focal-plane filters, which results in differential obscuration of up to a few percent; pixel-to-pixel variations in the CCD; "blobs" on the scale of a few tens of pixels across that may indicate contamination or may be artifacts of the CCD processing; and large-scale variations in sensitivity due to vignetting in the telescope optical system. So far, these patterns appear to be reasonably stable, and the RMS variation in the flat-field is of order $2 \%$. We will continue to monitor the flat-field through monthly secondary-mirror offpoints, and occasional spacecraft-offpoint maneuvers.

\subsection{Optical Performance}

The telescope mirrors were integrated into the tube assembly and aligned at SAO using a visible-light interferometer (Podgorski et al., 2009). The focal-plane assembly was integrated to the tube assembly at LMSAL. Optical performance of the integrated telescope assembly was checked at visible wavelengths using an infinite collimator to project a resolution target into the aperture. While this procedure allowed for an accurate determination of the position of best focus and could verify the absence of gross errors in the telescope's alignment or imaging performance, the visible-light measurements (using wavelengths $\approx 30 \times$ 

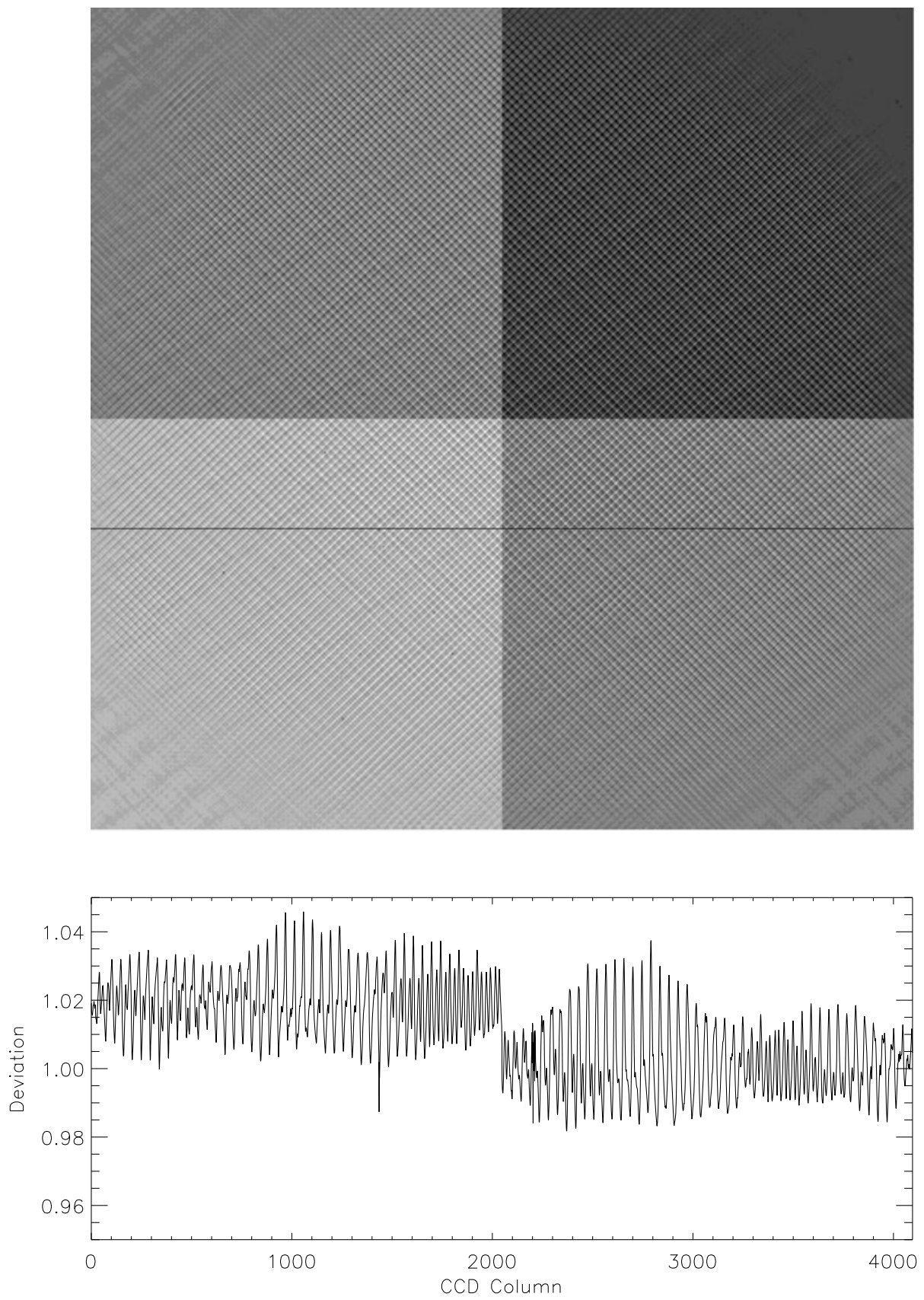

Figure 13 The flat-field of the 171-A channel. The dark line indicates the position of the cut, plotted below the image. 
Table 7 A sample image-resolution error budget for the $171-\AA ̊$ channel. The effects are added in quadrature to give an estimate of the system-level optical performance.

\begin{tabular}{ll}
\hline Item & $\begin{array}{l}\text { Contribution to } \\
\text { RMS Spot diameter } \\
{[\operatorname{arcsec}]}\end{array}$ \\
\hline Optical prescription & 0.60 \\
Fabrication, alignment, and assembly effects & 1.21 \\
Launch shift effects & 0.10 \\
On-orbit thermal effects & 0.21 \\
Focus error (with on-orbit correction) & 0.10 \\
Jitter residual & 0.48 \\
Diffraction (for UV-Vis channels) & 0.00 \\
Detector Pixelization & 0.48 \\
CCD Charge spreading & 0.80 \\
On-orbit performance Prediction & 1.73 \\
\hline
\end{tabular}

\begin{tabular}{lrl}
\cline { 2 - 2 } $\begin{array}{l}\text { Table } 8 \text { Predicted resolution } \\
\text { (RMS spot diameter in arcsec) }\end{array}$ for the AIA images. & Channel & Predicted Resolution \\
\cline { 2 - 3 } & $94 \AA$ & 1.74 \\
$131 \AA$ & 1.67 \\
$171 \AA$ & 1.73 \\
$193 \AA$ & 1.38 \\
$211 \AA$ & 1.55 \\
$304 \AA$ & 1.52 \\
$335 \AA$ & 1.73 \\
$1600 \AA$ & 1.65 \\
$1700 \AA$ & 1.65 \\
& $4500 \AA$ & 2.43 \\
\hline
\end{tabular}

Table 8 Predicted resolution (RMS spot diameter in arcsec) for the AIA images.

longer than the telescope's operational wavelengths) are limited by diffraction. They did not allow an accurate measurement of the in-band point-spread function.

The telescope spatial resolution was determined analytically by combining componentlevel interferometric measurements of the mirror figures with a raytrace model of the telescope prescription (including tolerances for mirror misalignment and structural and thermal deformations on orbit). Allocations for jitter, CCD pixelization, and charge spreading, diffraction (significant only for the UV-visible channels), and scatter by the mirror surfaces and the filter-support meshes were included as well. A minimal amount of defocus was assumed; the telescopes all have focus mechanisms that work by translating the secondary mirror along the optical axis, and on-orbit measurements have shown the focus to be stable. The stackup of these allocations for the 171- $\AA$ channel is shown in Table 7, and the predicted RMS spot diameters for all channels are listed in Table 8 . Note that the metric used in these tables is the RMS spot diameter, averaged over the field of view. This is not the limit of the spatial resolution of the instrument; there is significant contrast transfer at finer angular scales than those listed in Table 8. 


\subsubsection{Filter Mesh Diffraction}

The entrance-filter mesh creates a diffraction pattern, which is particularly noticeable for bright, compact sources such as flare kernels. The entrance filter for each AIA EUV channel consists of two independently-mounted mesh segments. In order to ensure that the diffraction patterns from the two filter segments can be separated from each other and from the solar image, the mesh grid of one segment is oriented at about $40^{\circ}$ with respect to the CCD columns, and the other is at about $50^{\circ}$. This results in an eight-legged diffraction pattern. The spacing of the maxima along each leg is given by

$$
\sin (\theta)=\frac{m \lambda}{a},
$$

where $\theta$ is the diffraction angle, $m$ is the order and $a$ is the mesh spacing. The mesh period is 70 lines per inch; the bars of the mesh cells are 29-33 microns thick.

The effect of diffraction by an entrance-filter mesh has been analyzed extensively for the TRACE entrance filters (Lin, Nightingale, and Tarbell, 2001; Gburek, Sylwester, and Martens, 2006). Similar techniques will be applied to the AIA images to characterize the mesh diffraction. The focal-plane filters use the same mesh, and therefore diffract light by the same mechanism; however, because they are so much closer to the CCD, the effect of this diffraction is much less significant. Instead, as previously discussed, they produce a screen-like shadow that is removed by flat fielding.

The telescope PSF, including the core (defined by the terms in Table 6) and the wings (which are dominated by the filter diffraction pattern), can be measured on orbit using the technique of DeForest, Martens, and Wills-Davey (2009), or by analysis of bright flare kernels. Once an accurate PSF is obtained, we will produce higher level data products with the PSF deconvolved out of the image.

\subsubsection{Stray Light}

The EUV entrance filters are routinely monitored for leakage of visible light by taking images without a focal-plane filter in place. The $\mathrm{Zr}$ entrance filters on the $94-\AA$ and $131-\AA$ channels transmit enough visible light to form a good-quality image of the photosphere in a $500-\mathrm{msec}$ exposure. The combination of the entrance filters and the focal-plane filters provides excellent rejection of out-of-band light for the EUV channels, and EUV light does not scatter efficiently off the interior surfaces of the telescope, so there is no substantial stray light in the EUV images.

The UV-channel images do show a significant ghost image approximately 2 arcmin to the Southeast of the main image. This is most likely due to internal reflections within the UV filter substrate. It may be possible to correct for the ghost image in the data processing pipeline.

\subsection{Guide Telescope-ISS}

Each ATA has a guide telescope (GT) rigidly mounted to it, which provides continuous measurements of the offset between the sunvector and the telescope boresight in pitch and yaw. This information is used by the telescope's image-stabilization system (ISS) to control the deflection of the active secondary mirror in order to cancel image motion or blurring due to spacecraft jitter. It is also sent to the spacecraft attitude control system (ACS) and used to maintain the observatory's pointing at the Sun. 
The ISS is an open-loop system in the sense that the corrections applied by the active secondary mirror are not directly sensed by the GT. Therefore, it relies on careful calibration of the relationship between the sunvector reported by the GT and the image deflection applied by the ISS electronics. The ISS calibration varies seasonally (due to changes in the apparent size of the solar disk, which changes the amplitude of the GT signal for a given angular deflection of the sunvector). Therefore, we perform monthly calibration maneuvers by offpointing the observatory in a cruciform pattern and measuring the response of the GT signals and the active secondary-mirror deflection at each position.

The image-stabilization system achieves a reduction of jitter by a factor of $>10$ for frequencies below $\approx 5 \mathrm{~Hz}$, and provides some attenuation for jitter up to $\approx 20 \mathrm{~Hz}$. This eliminates the impact from most of the dominant sources of jitter on SDO: the slow, thermallydriven drift in spacecraft pointing following eclipses, the stiction due to zero-speed crossings of the reaction wheels, and the torques of the AIA mechanisms themselves. There is some higher-frequency jitter (primarily due to the stepping of the high-gain antennas) which may introduce image blur at the level of a few tenths of an arcsecond for occasional exposures.

Differential shifts between the GT and the science telescope (ST) will cause pointing errors in the science data. This is generally not an issue, as the GT is rigidly coupled to the ST. However, during the first month of operations, the ISS introduced a slow $(\approx$ three five minutes) oscillation of $\approx 1$ arcsec amplitude as the cycling of the telescope heaters drove a differential pointing shift between the GT and ST. After this effect was discovered, the heater operations were tuned to maintain a stable temperature without cycling; since then, the pointing of all AIA channels has been extremely stable (less than 0.1 pixels of image motion on timescales of tens of minutes). Some image shift (up to a few pixels) due to thermal variations on longer time scales (days to months) persists, and there are small changes in pointing whenever the focus position of a channel is adjusted. These drifts will be corrected out as the operations and image processing techniques mature.

\subsection{Image Coalignment}

During integration, the four telescope assembly boresights were aligned to within 20 arcsecs of a common reference frame, and they maintained that coalignment through launch. The GTs were aligned to within 45 arcsecs of their respective STs; the GT boresights can effectively be shifted by up to 90 arcsecs in the onboard electronics by applying a bias to the signal from the GT limb sensors. Because the SDO ACS uses the GT signals for fine guiding, AIA is able to control the pointing of the observatory using the GT biases. The GT biases were chosen to coalign the four GTs, such that they all produce null signals when the spacecraft aligns the science reference boresight (SRB) (defined as the mean of the extreme science telescope boresights in pitch and yaw) with the Sun vector. This combined system has shown excellent stability, consistently maintaining science pointing to within a fraction of an arcsecond.

DC offsets of up to 15 arcsecs were applied to the active secondary mirrors of the telescopes to coalign each ST with the SRB. This reduced the misalignment of the science telescopes to a few arcseconds - thus, the center of the solar disk is within $\approx$ five pixels of the center of the CCD on all AIA channels before applying corrections in ground software. The CCDs are coaligned in roll to within \pm 0.25 degrees.

The residual misalignments between the channels have been measured using limb-fitting software and "manual" comparison of bright features on the disk and at the limb. The alignment information is documented in the image headers at Level 1, and corrected out of the image at Level 1.5. 

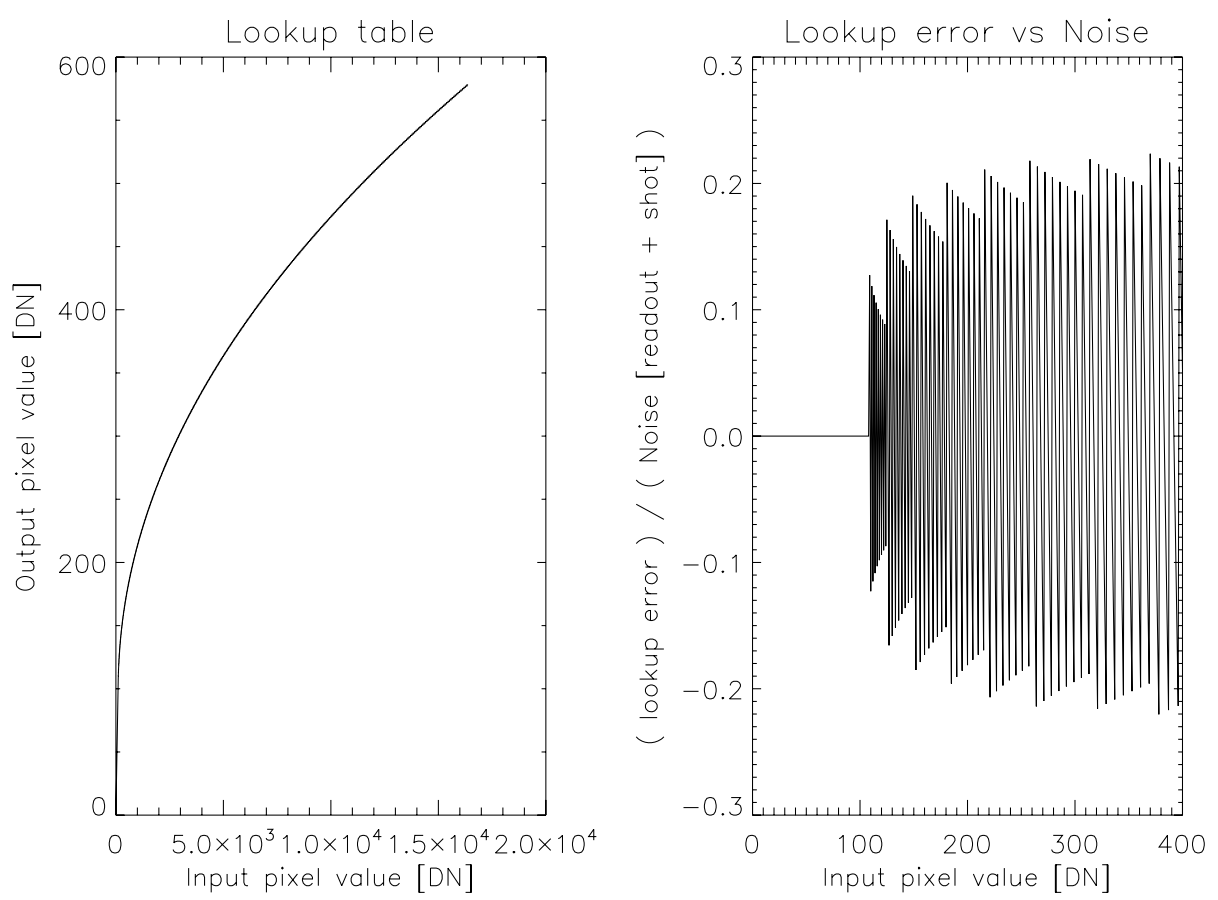

Figure 14 Left: An example onboard lookup table used for lossy compression. Right: The ratio of the error in pixel values due to the lookup table to the intrinsic error in the measurement (due to shot noise and readout noise).

\subsection{Compression}

AIA downlinks science data at a higher rate than any solar science instrument that has ever flown. Its telemetry allocation is capped at $67 \mathrm{Mbps}$; the observing sequence is designed to fill $80-90 \%$ of this bandwidth all the time. In order to maintain a cadence of 10 or 12 seconds for full-frame images from all eight AIA channels while fitting within this bandwidth, it is necessary to perform some compression onboard. Without compression, the eight channel-by-ten second cadence generates $4096 \times 4096 \times 16 \times 8 / 10=215 \mathrm{Mbps}$ of data, so compression of approximately a factor of four is required (to approximately four bits per pixel). This level of compression cannot be reliably achieved without using slightly lossy compression techniques.

The image data are compressed by an onboard FPGA using a Rice compression algorithm (Rice, 1979) that converts the pixel values into differences between adjacent pixels. Small differences compress extremely efficiently (down to a limit of two bits per pixel for very smoothly varying images), while sharp intensity variations (especially due to cosmic-ray hits or bright coronal features) require much more data to transmit. The Rice algorithm itself is lossless; however, in order to maximize its effectiveness, it is necessary to minimize the impact of large intensity variations. This is accomplished by remapping the image pixel values using a lookup table before applying the Rice compression. A sample lookup table is shown in Figure 14. Note that the difference between the "true" pixel value and the value that it is rounded off to (the error introduced by the compression scheme) is approximately proportional to the square root of the true pixel value - in this case, it is always less than $1 / 4$ 
of the value. This ensures that the compression error will be small compared to the intrinsic shot noise in the image for all types of observations. The images are decompressed during the production of level 0 data (Lemen et al., 2011), and therefore the data user does not need to make any corrections for the compression.

\section{Conclusion}

The preflight photometric calibration of the AIA instrument shows good agreement with absolute irradiance measurements by the EVE rocket and with theoretical intensity predictions from CHIANTI. The response functions will be updated within the first year of operations based on cross-calibrations with EVE and closer analysis of the AIA images themselves.

The preliminary on-orbit characterization of the instrument performance is complete, and, where possible, corrections for instrumental effects (such as flat-fielding, image offsets, etc.) have been applied to the calibrated data. The instrument is healthy and in good working order, and should continue to provide excellent data for years to come.

Acknowledgements This work is supported by NASA under contract NNG04EA00C.

Open Access This article is distributed under the terms of the Creative Commons Attribution Noncommercial License which permits any noncommercial use, distribution, and reproduction in any medium, provided the original author(s) and source are credited.

\section{Appendix: Accessing the Response Functions}

The instrument-wavelength response and temperature-response functions are available through the SolarSoft (SSW) IDL library (Freeland and Handy, 1998), using the function aia_get_response. The wavelength-response functions are retrieved as follows: IDL> aia_resp = aia_get_response(/area [, /dn, /full, /uv, /all]) where the optional keyword /dn specifies that the photon-to-DN conversion should be included; /full indicates that the detailed component-level calibration results (including, e.g., the mirror reflectivity, CCD QE, etc.) should be returned; /uv selects the UV and visible channels rather than the (default) EUV channels; and /all indicates that responses for both thin and thick focal-plane filter positions should be returned.

The default solar emissivity, calculated using CHIANTI 6.0.1, can be returned as follows: IDL > aia_emiss = aia_get_response(/emissivity [,/full]) where the optional keyword /full will return the detailed line list.

The temperature-response functions of the EUV channels can be accessed as follows: IDL $>$ aia_tresp = aia_get_response(/temp [, /full, /dn, /all]) where the optional keywords /dn and /all have similar meanings to those used with the effective area, and /full in this context returns the response for each channel as a function of both wavelength and temperature. The temperature response is calculated on demand, using effective area and emissivity data that are stored in the SSW database.

A number of lower-level routines (with filenames beginning with aia_bp_) are also available in SSW, allowing the user to adjust or apply the response functions (e.g. to calculate count rates given an input spectrum, generate a temperature-response function using alternate assumptions about ionization balance, etc.). 


\section{References}

Catura, R.C., Stern, R.A., Cash, W., Windt, D.L., Culhane, J.L., Lappington, J., Barnsdale, K.: 1987, Proc. SPIE 830, 204.

DeForest, C.E., Martens, P.C.H., Wills-Davey, M.J.: 2009, Astrophys. J. 690, 1264.

Dere, K.P., Landi, E., Mason, H.E., Monsignori Fossi, B.C., Young, P.R.: 1997, Astron. Astrophys. Suppl. $125,149$.

Dere, K.P., Moses, J.D., Delaboudinière, J.-P., Brunaud, J., Carabetian, C., Hochedez, J.-F., Song, X.Y., Catura, R.C., Clette, F., Defise, J.-M.: 2000, Solar Phys. 195, 13.

Dere, K.P., Landi, E., Young, P.R., Del Zanna, G., Landini, M., Mason, H.E.: 2009, Astron. Astrophys. 498, 915.

Feldman, U., Widing, K.G.: 1993, Astrophys. J. 414, 381.

Freeland, S.L., Handy, B.N.: 1998, Solar Phys. 182, 497.

Gburek, S., Sylwester, J., Martens, P.: 2006, Solar Phys. 239, 531.

Handy, B.N., Acton, L.W., Kankelborg, C.C., Wolfson, C.J., Akin, D.J., Bruner, M.E., Caravalho, R., Catura, R.C., Chevalier, R., Duncan, D.W., Edwards, C.G., Feinstein, C.N., Freeland, S.L., Friedlaender, F.M., Hoffmann, C.H., Hurlburt, N.E., Jurcevich, B.K., Katz, N.L., Kelly, G.A., Lemen, J.R., Levay, M., Lindgren, R.W., Mathur, D.P., Meyer, S.B., Morrison, S.J., Morrison, M.D., Nightingale, R.W., Pope, T.P., Rehse, R.A., Schrijver, C.J., Shine, R.A., Shing, L., Strong, K.T., Tarbell, T.D., Title, A.M., Torgerson, D.D., Golub, L., Bookbinder, J.A., Caldwell, D., Cheimets, P.N., Davis, W.N., Deluca, E.E., McMullen, R.A., Warren, H.P., Amato, D., Fisher, R., Maldonado, H., Parkinson, C.: 1999, Solar Phys. 187, 229.

Henke, B.L., Gullikson, E., Davis, J.C.: 1993, Atom. Data Nucl. Data Tables 54(2), 181.

Hurlburt, N., Cheung, M., Schrijver, C., Chang, L., Freeland, S., Green, S., Heck, C., Jaffey, A., Kobashi, A., Schiff, D., Serafin, J., Seguin, R., Slater, G., Somani, A., Timmons, R.: 2011, Solar Phys. doi:10.1007/s11207-010-9624-2.

Judge, P.G., Hubeny, V., Brown, J.C.: 1997, Astrophys. J. 475, 275.

Kuhn, J.R., Lin, H., Loranz, D.: 1991, Publ. Astron. Soc. Pac. 103, 1097.

Lemen, J.R., Title, A.M., Akin, D.J., Boerner, P.F., Chou, C., Drake, J.F., Duncan, D.W., Edwards, C.G., Friedlaender, F.M., Heyman, G.F., Hurlburt, N.E., Katz, N.L., Kushner, G.D., Levay, M., Lindgren, R.W., Mathur, D.P., McFeaters, E.L., Mitchell, S., Rehse, R.A., Schrijver, C.J., Springer, L.A., Stern, R.A., Tarbell, T.D., Wuelser, J.-P., Wolfson, C.J., Yanari, C.: 2011, Solar Phys. doi:10.1007/s11207-011-9776-8

Lin, A.C., Nightingale, R.W., Tarbell, T.D.: 2001, Solar Phys. 198, 385.

Podgorski, W.A., Cheimets, P.N., Boerner, P.F., Glenn, P.: 2009, Proc. SPIE 7438, 13.

Pottasch, S.R.: 1963, Astrophys. J. 137, 945.

Rice, R.F.: 1979, Some Practical Universal Noiseless Coding Techniques, JPL Publication 79-22, Pasadena, CA.

Soufli, R., Baker, S.L., Windt, D.L., Gullikson, E.M., Robinson, J.C., Podgorski, W.A., Golub, L.: 2007, Appl. Opt. 46, 3156.

Soufli, R., Windt, D.L., Robinson, J.C., Baker, S.L., Spiller, E., Dollar, F.J., Aquila, A.L., Gullikson, E.M., Kjornrattanawanich, B., Seely, J.F., Golub, L.: 2005, Proc. SPIE 5901, 173.

Stern, R.A., Shing, L., Blouke, M.: 1994, Appl. Opt. 33, 2521.

Stern, R.A., Shing, L., Catura, P., Morrison, M., Duncan, D., Lemen, J., Eaton, T., Pool, P., Steward, R., Walton, D., Smith, A.: 2004, Proc. SPIE 5171, 77.

Sylwester, J., Schrijver, J., Mewe, R.: 1980, Solar Phys. 67, 285.

Waltham, N., Beardsley, S., Clapp, M., Lang, J., Jerram, P., Pool, P., Auker, G., Morris, D., Duncan, D.: 2011, In: Internat. Conf. Space Optics, ESA, Noordwijk, in press.

Warren, H.: 2005, Astrophys. J. Suppl. 157, 147.

Windt, D.: 1998, Comput. Phys. 12, 4 (), 360.

Windt, D., Catura, R.: 1988, Proc. SPIE 984, 82.

Withbroe, G.L.: 1975, Solar Phys. 45, 301.

Woods, T.N., Eparvier, F.G., Bailey, S.M., Chamberlin, P.C., Lean, J., Rottman, G.J., Solomon, S.C., Tobiska, W.K., Woodraska, D.L.: 2005, J. Geophys. Res. 110, 01312.

Woods, T.N., Woods, T.N., Chamberlin, P., Eparvier, F.G., Hock, R., Jones, A., Woodraska, D., Judge, D., Didkosky, L., Lean, J., Mariska, J., McMullin, D., Warren, H., Berthiaume, G., Bailey, S., Fuller-Rowell, T., Sojka, J., Tobiska, W.K., Viereck, R.: 2010, Solar Phys. doi:10.1007/s11207-009-9487-6. 